\title{
Sustained Release of Protein Therapeutics from Subcutaneous Thermosensitive Biocompatible and Biodegradable Pentablock Copolymers (PTSgels)
}

\author{
Elizabeth Schaefer, ${ }^{1}$ Santhi Abbaraju, ${ }^{2}$ Mary Walsh, ${ }^{1}$ Donna Newman, ${ }^{1}$ Jacklyn Salmon, ${ }^{1}$ \\ Rasidul Amin, ${ }^{2}$ Sidney Weiss, ${ }^{3}$ Ulrich Grau, ${ }^{3}$ Poonam Velagaleti, ${ }^{3}$ and Brian Gilger ${ }^{1}$ \\ ${ }^{1}$ North Carolina State University, Raleigh, NC, USA \\ ${ }^{2}$ Symmetry Biosciences, Research Triangle Park, NC, USA \\ ${ }^{3} i$-novion, Inc., Randolph, NJ, USA
}

Correspondence should be addressed to Brian Gilger; bgilger@ncsu.edu

Received 16 May 2016; Accepted 25 July 2016

Academic Editor: Kang Choon Lee

Copyright (c) 2016 Elizabeth Schaefer et al. This is an open access article distributed under the Creative Commons Attribution License, which permits unrestricted use, distribution, and reproduction in any medium, provided the original work is properly cited.

\begin{abstract}
Objective. To evaluate thermosensitive, biodegradable pentablock copolymers (PTSgel) for sustained release and integrity of a therapeutic protein when injected subcutaneously. Materials and Methods. Five PTSgels with PEG-PCL-PLA-PCL-PEG block arrangements were synthesized. In vitro release of IgG from PTSgels and concentrations was evaluated at $37^{\circ} \mathrm{C}$. Released IgG integrity was characterized by SDS-PAGE. In vitro disintegration for $10 \mathrm{GH}$ PTSgel in PBS was monitored at $37^{\circ} \mathrm{C}$ over 72 days using gravimetric loss and GPC analysis. Near-infrared IgG in PTSgel was injected subcutaneously and examined by in vivo imaging and histopathology for up to 42 days. Results. IgG release was modulated from approximately 7 days to more than 63 days in both in vitro and in vivo testing by varying polymer composition, concentration of PTSgel aqueous solution, and concentration of IgG. Released IgG in vitro maintained structural integrity by SDS-PAGE. Subcutaneous PTSgels were highly biocompatible and in vitro IgG release occurred in parallel with the disappearance of subcutaneous gel in vivo. Conclusions. Modulation of release of biologics to fit the therapeutic need can be achieved by varying the biocompatible and biodegradable PTSgel composition. Release of IgG parallels disappearance of the polymeric gel; hence, little or no PTSgel remains after drug release is complete.
\end{abstract}

\section{Introduction}

Increase in the use of biologics has occurred over the past decade for the treatment, prevention, or cure of a variety of diseases in humans. Biological products include blood-derived products, vaccines, immunoglobulins, cells or microorganisms, and other proteins [1-4]. These have led to major therapeutic advances in several prevalent diseases, including immune-mediated arthritis and cancer immunotherapy [5]. Many biologics are administered to the patient, usually by daily or weekly subcutaneous injection. A controlled, sustained release therapeutic would decrease the frequency of injections, leading to increased patient compliance and therapeutic efficacy.
Sustained release subcutaneous therapeutics have been available for several decades, but recent advances in polymer science have led to development of hydrogels that provide sustained drug release, have high tissue biocompatibility, and allow self-administration by the patient [6]. Hydrogels provide a deformable drug depot that slowly elutes a high concentration of drug to surrounding tissue for an extended period of time [6]. However, because most hydrogels only physically incorporate, instead of forming covalent bonds to the drugs, a rapid drug release occurs over a few hours to days, limiting their value for sustained drug delivery [6].

Triblock copolymers of poly(ethylene oxide)-poly(propylene oxide)-poly(ethylene oxide) (PEO-PPO-PEO, poloxamers/pluronics) are the most widely used reverse thermal 
gelation polymers [7]. Other types of multiblock amphiphiles (i.e., polymers with both hydrophilic and hydrophobic domains) have been synthesized using a wide range of polymers. Some of these hydrogels are sufficiently deformable to be injectable, but many are not, necessitating surgical implantation for drug delivery. In either case, a high initial burst and lack of sustained drug release limit the clinical utility of these hydrogels $[6,8]$.

Polylactic-co-glycolic acid (PLGA) based hydrogels exhibit better biodegradability, higher gelation temperatures (permitting easier handling before injection), and longer periods of sustained drug release compared to poloxamer systems [9]. However, degradation of PLGA and PLGA copolymers produces lactic acid and glycolic acid, which reduces local $\mathrm{pH}$ substantially and may degrade protein therapeutics [10]. Furthermore, local tissue reaction to the PLGA may reduce tolerability and biocompatibility [11]. Therefore, an injectable and biocompatible hydrogel that provides a sustained release of biologically active protein therapeutic remains to be developed.

Pentablock copolymers are thermosensitive gels (PTSgel) composed of biodegradable or biocompatible polymer blocks, such as polyethylene glycol (PEG), polycaprolactone (PCL), polyglycolide (PGA), and polylactic acid (PLA). The relative block arrangement and molecular weight of PTSgel polymers affect the solution-gelation (sol-gel) transition behavior, degradation, and in vitro release characteristics of the hydrogel [12]. PTSgels may act as a drug delivery vehicle by entrapping the drug in the core of a micelle of PTSgel [12]. PTSgels can be injected through a small-gauge needle to form a firm, in situ, hydrogel depot [12]. PTSgels have been demonstrated to be biocompatible in vitro and in vivo and provide sustained release of immunoglobulin $\mathrm{G}$ $(\operatorname{IgG})[12,13]$. Furthermore, enhanced stability of biologic proteins (IgG and bevacizumab) delivered from PTSgels in vitro was recently shown [13]. The amounts of PLA used in the described polymers ranged from 28 to $37 \%$ of the total molar mass. Compared to PLGA, the lower molar mass of PLA or PGA blocks in the PTSgel produces much lower amounts of lactic acid or glycolic acid on degradation, thereby improving protein stability of the delivered biologic. Therefore, the potential advantages of PTSgels as carriers for subcutaneous sustained delivery of protein biologic therapeutics include biodegradation, their high biocompatibility, long-term release kinetics, ease of injectability, and stability of the protein therapeutic being delivered.

The objective of this work was to further evaluate the sustained release properties of promising thermosensitive PTSgels for the controlled release of a model full-length therapeutic protein (IgG; mw $150 \mathrm{kDal}$ ) for subcutaneous injection. This study investigated the in vitro modulated release of IgG, the structural integrity of released IgG, and the in vivo duration of IgG release from PTSgel after subcutaneous injection. In vitro/in vivo correlation has been established and presented for selected PTSgel polymers. The study also investigated in vitro disintegration of $10 \mathrm{GH}$ PTSgel in PBS (pH 7.4) at $37^{\circ} \mathrm{C}$ over a period of several weeks.

\section{Materials and Methods}

2.1. PTSgel Synthesis. Monomethoxy PEG (550), L-lactide, tin octoate, hexamethylenediisocynate (HMDI), ethylene glycol-bis(2-aminoethylether)-N,N,N ${ }^{\prime} \mathrm{N}^{\prime}$-tetraacetic acid (EGTA), sodium sulfate, dichloromethane, heptanes, and endotoxin free water were purchased from Sigma-Aldrich (St. Louis, MO). $\varepsilon$-Caprolactone was purchased from Alfa Aesar (Ward Hill, MA). PTSgels with PEG-PCL-PLA-PCL-PEG block arrangements were synthesized as previously described $[12,13]$. Briefly, the diblock copolymer was synthesized by ring-opening copolymerization of $\varepsilon$-caprolactone with monomethoxy PEG using tin octoate as a catalyst. The resulting diblock copolymer was similarly converted to triblock by adding L-lactide. The resulting triblock copolymer was coupled utilizing hexamethylenediisocynate (HMDI) as a linker to prepare PEG-PCL-PLA-PCL-PEG pentablock copolymers. The purified pentablock was stored at $-20^{\circ} \mathrm{C}$ until being used.

\subsection{PTSgel Characterization}

2.2.1. Fourier Transform Infrared Spectroscopy (FTIR) Analysis. Fourier transform infrared spectroscopy (FTIR) spectra were recorded with a Perkin Elmer Spectrum Version 10.03.09 infrared spectrophotometer. FTIR scan of neat polymer was carried out in a range of $4000-400 \mathrm{~cm}^{-1}$.

2.2.2. ${ }^{1} H$-NMR Analysis. Purity, molecular structure, and molecular weight $(\mathrm{Mn})$ of the PTSgel were analyzed utilizing a Mercury $300 \mathrm{MHz}$ NMR spectrometer. ${ }^{1} \mathrm{H}-\mathrm{NMR}$ spectrograms were recorded by dissolving the polymers in deuterated chloroform $\left(\mathrm{CDCl}_{3}\right)$.

2.2.3. Gel Permeation Chromatography (GPC) Analysis. Molecular weights ( $\mathrm{Mn}$ and $\mathrm{Mw}$ ) and polydispersity of polymers were examined by GPC analysis. Briefly, $20 \mathrm{mg}$ of polymer was dissolved in $1 \mathrm{~mL}$ of tetrahydrofuran (THF). Polymer samples were separated on two OligoPore columns (Agilent, Santa Clara, CA) connected in series and maintained at $40^{\circ} \mathrm{C}$. Solvent THF at the rate of $0.6 \mathrm{~mL} / \mathrm{min}$ was utilized as eluting solvent. Samples were analyzed on Wyatt technologies MINI DAWN instrument (S. number 528-T) connected to OPTILAB DSP interferometric refractometer, using ASTRA 6 software.

2.2.4. Determination of Tin, Organic Solvents, and Endotoxin Levels in the Polymers. Amount of stannous (Tin) present was determined on $5 \%$ aqueous polymer solution using ICPOES method on a Thermo iCAP 6100 instrument. Residual dichloromethane and heptane in the PTSgel were determined by head space GC-MS using an Agilent 5890 GC and an Agilent 5972 mass spectrometer. The column employed was Restek Rtx-5MS, $30 \mathrm{~m} \times 0.25 \mathrm{~mm}, 0.1 \mu \mathrm{m}$ film. The endotoxin levels were determined using Pierce Chromogenic Endotoxin Quantification Kit (Thermo Fisher Scientific, Waltham, MA). 
2.3. Sol-Gel Transition Testing at $37^{\circ} \mathrm{C}$. The sol (flow)-gel (no flow) transition of PTSgel was examined by following a previously published protocol [14]. Briefly, the polymers were dissolved in PBS buffer ( $\mathrm{pH} 7.4)$ at $25 \mathrm{wt} \%$ concentration. $0.5 \mathrm{~mL}$ of aqueous polymeric solution was transferred into $2.5 \mathrm{~mL}$ glass vial and placed in water bath maintained at $37^{\circ} \mathrm{C}$. Vials were kept for $5 \mathrm{~min}$ at $37^{\circ} \mathrm{C}$. Gel formation was observed visually by inverting the tubes, immediately after pulling out of the water bath.

\subsection{In Vitro PTSgel Disintegration (Incubation in PBS Buffer, pH 7.4 at $\left.37^{\circ} \mathrm{C}\right)$}

2.4.1. Gravimetric Measurement of the Residual Gelling Polymer. Three concentrations $(12.5,18.75$, and 25\%) of PTSgel $10 \mathrm{GH}$ were evaluated for disintegration/degradation in vitro. Each individual PTSgel $(500 \mu \mathrm{L}$ of $10 \mathrm{GH}$ in triplicate) was pipetted into an $8 \mathrm{~mL}$ glass vial, weighed, and placed into a $37^{\circ} \mathrm{C}$ water bath for gelling for 30 minutes. $4 \mathrm{~mL}$ of PBS $\left(37^{\circ} \mathrm{C}\right)$ with $0.02 \%$ sodium azide as preservative was placed over each PTSgel. The vials were placed into a $37^{\circ} \mathrm{C}$ shaker water bath, maintained at $60 \mathrm{rpm}$. Every 5 days, vials were centrifuged ( $2000 \mathrm{rpm}$ for 5 minutes at $37^{\circ} \mathrm{C}$ ), the PBS buffer was removed and replaced with fresh PBS, and the vials returned to the $37^{\circ} \mathrm{C}$ shaker water bath. Samples of gel were withdrawn after $0,5,15,30$, and 45 days and every 15 days thereafter until polymer completely disintegrated. At each time point, vials with residual gel were stored at $-80^{\circ} \mathrm{C}$ until being lyophilized. Vials containing lyophilized gels were weighed and the dry gel weight was determined by subtracting the empty vial weight from the final dry weight.

\subsubsection{GPC Analysis of the Residual Gelling Polymer and the} Supernatant. The residual gelling polymer (PTSgel 10GH) and the supernatant collected during the gravimetric studies were analyzed using GPC. The gels were lyophilized using a Labconco Model 5 lyophilizer (Kansas City, MO) and 10$50 \mathrm{~mL}$ of the buffer samples were dried using a Genevac EZ-2 evaporator overnight. The lyophilized and dried samples were dissolved in $1 \mathrm{~mL}$ of tetrahydrofuran and further dried using $0.3-0.5 \mathrm{~g}$ of sodium sulfate. Samples were then vortexed and filtered using $0.2 \mu \mathrm{m}$ PTFE ISO disc filters. Selected samples were analyzed by GPC as described in Section 2.2.3.

2.5. In Vitro Release of IgG from PTSgel. In vitro drug release experiments were conducted by adding $500 \mu \mathrm{L}$ of $22.5 \%$ aqueous gelling polymeric solution containing $10 \mathrm{mg}$ of IgG (Human IgG, Lee Biosolutions, Maryland Heights, MO) into an $8 \mathrm{~mL}$ silanized glass vial (Thermo Scientific, Waltham, MA) in triplicate. Vials were incubated in a $37^{\circ} \mathrm{C}$ water bath for $\sim 5$ minutes until polymer gelled. Release buffer, $4 \mathrm{~mL}$ of phosphate buffer saline (PBS, pH 7.4) with $0.02 \%$ (wt/vol) sodium azide, was gently layered over the solidified gel in each vial. Vials were sealed with parafilm and maintained at $60 \mathrm{rpm}$ in a $37^{\circ} \mathrm{C}$ water bath to replicate physiologic conditions. $2 \mathrm{~mL}$ out of the $4 \mathrm{~mL}$ of overlaid buffer solution was removed from each vial on days $1,2,3,4,5,7,10$, and 14 and then weekly thereafter until no more IgG was released.
Following collection, $2 \mathrm{~mL}$ PBS release buffer with $0.02 \%$ sodium azide (maintained at $37^{\circ} \mathrm{C}$ ) was layered back into the test vials which were returned to the shaker bath until the next sampling period. The concentration of released IgG samples was evaluated by comparing the released samples to a standard IgG calibration curve. Calibrators and release samples $(200 \mu \mathrm{L})$ were pipetted into a 96-well, UV-free microplate (Greiner Bio-One, Monroe, NC) in triplicate and the absorbance was measured at $280 \mathrm{~nm}$ (Synergy 2 Microplate Reader, BioTek, Winooski, VT). All experiments were set up in triplicate and absorbance of a blank control (0\% IgG in 22.5\% PTSgel) was subtracted from the released samples for estimation of protein concentration. Similar experiments were set up using three different concentrations of 10GH PTSgel to evaluate further modulation of the release profiles.

\subsection{After Releasing IgG Integrity}

2.6.1. Sodium Dodecyl Sulfate Polyacrylamide Gel Electrophoresis (SDS-PAGE) Analysis: Reducing and Nonreducing. Collected buffer samples from the in vitro release assays were evaluated for IgG integrity by SDS-PAGE analysis. The IgG samples included were standards and the samples released in PBS buffer $\left(\mathrm{pH} 7.4,37^{\circ} \mathrm{C}\right)$ after incorporation into a selected PTSgel. Samples were evaluated within 7 days of collection and stored at $4^{\circ} \mathrm{C}$ until analysis. IgG standards, diluted in PBS, or sample eluates were combined with $4 \mathrm{x}$ Laemmli dye, with (reducing) or without (nonreducing) $\beta$ mercaptoethanol, to achieve a $1 \mathrm{x}$ dye concentration. Reduced samples were heated to $95^{\circ} \mathrm{C}$ for 10 minutes, cooled, and loaded on $4-12 \%$ Bis-Tris NuPAGE gels (Life Technologies, Carlsbad, CA). Prestained markers (cat. number LC5925, Life Technologies) and nonreduced samples were loaded without heating. Gels were run in MOPS buffer (Life Technologies), with antioxidants for reduced samples only, at $180 \mathrm{~V}$ for 60 minutes. Electrophoresed gels were fixed in 50\% methanol, $10 \%$ acetic acid for 30 minutes, stained in Coomassie Brilliant Blue R250 Staining Solution (Bio-Rad Laboratories, Inc., Hercules, CA) for 30 minutes, and destained in 5\% methanol, $7.5 \%$ acetic acid until clearance. Gels were scanned on a Canon CanoScan 9000F at $600 \mathrm{dpi}$ and images saved as TIFFs in Adobe Photoshop.

2.6.2. Size Exclusion HPLC Analysis (Shodex Column). Collected buffer samples from the in vitro release assays were evaluated for IgG integrity by SE-HPLC analysis. The IgG samples included were standards and the samples released in PBS buffer $\left(\mathrm{pH} 7.4,37^{\circ} \mathrm{C}\right)$ after incorporation into a selected PTSgel for up to 28 days. Samples were stored at $-20^{\circ} \mathrm{C}$ until analysis. Twenty microliters of IgG standards, diluted in PBS, or sample eluates were analyzed on a Shodex column (KW403-4F, $4.6 \mathrm{~mm}$ ID $\times 300 \mathrm{~mm} \mathrm{~L}$ ) using a UV absorption detector with the wavelength selected at $214 \mathrm{~nm}$. The mobile phase was $100 \mathrm{mM}$ sodium phosphate, $250 \mathrm{mM}$ $\mathrm{NaCl}, \mathrm{pH} 7.0$, used under isocratic conditions at $0.35 \mathrm{~mL} /$ $\min$. 
2.7. In Vivo Subcutaneous Release of IgG in Mice. Use of animals in this study was approved and monitored by the North Carolina State University Institutional Animal Care and Use Committee (IACUC). IgG was labeled with a nearinfrared (NIR) dye (IRDye $800 \mathrm{CW}$ by LICOR Biosciences, Lincoln, NE). NIR-labeled IgG in PTSgel gelling solution or in PBS was made by adding $1 \mathrm{~mL}$ of cold PTSgel (25\% polymer solution in PBS, pH 7.4) or PBS to $1 \mathrm{mg}$ of lyophilized NIRlabeled IgG. After gentle vortexing, the solutions were stored at $4^{\circ} \mathrm{C}$ until being used within 24 hours. Insulin syringes with $31 \mathrm{G}$ needles were used to inject $200 \mu \mathrm{L}$ of solution subcutaneously over the dorsum of a female CD-1 mouse (10 weeks of age) (Charles River, Morrisville, NC) that was maintained on an alfalfa-free diet. Mice $(n=3)$ were injected with PBS, PTSgel, or a combination of NIR-IgG in PBS or NIR-IgG in PTSgel. Mice were anesthetized with 2.5\% isoflurane in oxygen and imaged using an in vivo imager (IVIS, Xenogen, Alameda, CA) using Indocyanine Green (ICG) settings. Quantification of fluorescence was measured using the imaging software automatic region of interest (ROI) setting to calculate the radiant efficiency of the injection site. Mice were imaged prior to injection, immediately after injection, and then on days $1-5,7,10$, and 14 after injection and then weekly using the same imager settings and protocol as used for day 0 imaging.

2.8. In Vivo Safety Assessment. Assessment of the injection site was done at each imaging time to evaluate for signs of inflammation or swelling. Once the injection site was negative for dye detection on IVIS imaging, the mice were euthanized and the skin at the injection site was collected, the inverted skin exposing the injection site/PTSgel depot was imaged ex vivo using IVIS imaging to detect residual IgG, and the skin section was fixed in $10 \%$ formalin. The formalin-fixed skin was then processed for histopathology, stained with hematoxylin and eosin, and examined using light microscopy.

\section{Results}

3.1. PTSgel Synthesis. For the purposes of this study, five thermosensitive PTSgel polymers were synthesized. The five polymers developed for this study were designated 101GH, $10 \mathrm{GH}, 103 \mathrm{GH}, 113 \mathrm{GH}$, and $122 \mathrm{GH}$. The polymers were constructed with different block sizes of m-PEG, PCL, and PLA with PLA in the center of the molecule (m-PEG ${ }_{x}-\mathrm{PCL}_{y^{-}}$ $\left.\mathrm{PLA}_{z}-\mathrm{PCL}_{y}-\mathrm{PEG}_{x}-\mathrm{m}\right)$. The molecular weight $(\mathrm{Mw})$ ranged between 3000 and $4000 \mathrm{Da}$ with gradual increase in the hydrophobicity of molecules. The objective was to vary molecular weights and hydrophobic-hydrophilic block ratios in the polymers to achieve modulation of drug release. In the described polymers, the PEG blocks were $\mathrm{Mw}$ of 500 or $550 \mathrm{Da}$, the PCL block Mw ranged from 400 to $800 \mathrm{Da}$, and the PLA block was of constant size at Mw 1100 across all polymers. All polymers were characterized by NMR, FTIR for structural confirmation, and GPC for PDI determination and ability to transition from liquid phase to gel at $37^{\circ} \mathrm{C}$. All five polymers were compared for in vitro release profiles. Two polymers, $10 \mathrm{GH}$ and $113 \mathrm{GH}$, were used for in vivo subcutaneous release, polymer disappearance, and safety investigations. $10 \mathrm{GH}$ at various concentrations was also analyzed for in vitro degradation analyses.

\subsection{PTSgel Characterization}

FTIR Spectrum. FTIR spectrum of $10 \mathrm{GH}$ polymer is reported in Figure 1(a). Absorption band at $1726 \mathrm{~cm}^{-1}$ and multiple bands ranging from 1000 to $1300 \mathrm{~cm}^{-1}$ established the presence of ester linkages in pentablock copolymer. Existence of terminal hydroxyl group was confirmed by $\mathrm{C}-\mathrm{O}$ stretching band at $1092 \mathrm{~cm}^{-1}$ and $\mathrm{O}-\mathrm{H}$ band (stretch) in the range of $3300-3400 \mathrm{~cm}^{-1}$. C-H stretching bands at 2938 and $2866 \mathrm{~cm}^{-1}$ depicted presence of PCL blocks. The presence of $\mathrm{N}-\mathrm{H}$ (stretch) in the urethane group may be overlapped in the area of $\mathrm{O}-\mathrm{H}$ band (stretch) $3300-3400 \mathrm{~cm}^{-1}$. Similarly, the $\mathrm{C}=\mathrm{O}$ in urethane group might have been overlapped with other $\mathrm{C}=\mathrm{O}$ (stretch) peaks from ester linkages in pentablock copolymer. Absorption band at $1529 \mathrm{~cm}^{-1}$ (N-H bending) also exhibited the presence of urethane group in pentablock copolymer.

${ }^{1} H$-NMR Spectrum. A Mercury $300 \mathrm{MHz}$ NMR spectrometer was employed to characterize the pentablock copolymers. Figure 1(b) depicts ${ }^{1} \mathrm{H}-\mathrm{NMR}$ spectra of $10 \mathrm{GH}$ in deuterated chloroform. As described in Figure 1(b), typical ${ }^{1} \mathrm{H}$ NMR characteristic peaks were observed at 1.55, 2.30, and $4.04 \delta \mathrm{ppm}$ representing methylene protons of $-\left(\mathrm{CH}_{2}\right)_{3}^{-}$, $\mathrm{OCOCH}_{2}^{-}$, and $-\mathrm{CH}_{2} \mathrm{OOC}$ - of PCL units, respectively. A sharp peak at $3.64 \delta$ ppm was attributed to methylene protons $\left(-\mathrm{CH}_{2} \mathrm{CH}_{2} \mathrm{O}-\right)$ of PEG. Typical signals at $1.50\left(-\mathrm{CH}_{3}\right)$ and 5.17 (-CH-) $\delta$ ppm were assigned for PLA blocks, whereas a peak at $3.36 \delta \mathrm{ppm}$ was denoted to terminal methyl $\left(-\mathrm{OCH}_{3}^{-}\right)$of PEG. The [EO]-[CL]-[LA] molar ratios of final products were calculated from integrations of PEG signal at $3.36 \delta \mathrm{ppm}$, PCL signal at $4.04 \delta \mathrm{ppm}$, and PLA signal at $5.17 \delta \mathrm{ppm}$. PEG signal at $3.36 \delta$ ppm was applied for the calculation of molar ratio of various blocks within the pentablock copolymer. Estimated molecular weight, calculated using NMR, was close to theoretical feed ratio (Table 1).

Molecular Weight (Mw and Mn) and Polydispersity Determination. Molecular weight ( $\mathrm{Mw}$ and $\mathrm{Mn}$ ) and polydispersity of polymers were determined by GPC. Retention time for the three components used for PTSgel synthesis is depicted in Figure 1(c). A typical GPC chromatogram of $10 \mathrm{GH}$ pentablock copolymer is shown in Figure 1(d). A single peak for the polymer was observed describing unimodal distribution of molecular weight and absence of any other homopolymer block such as PEG, PCL, or PLA. Polydispersity (PDI) for the five analyzed polymers ranged from 1.08 to 1.28 indicating narrow distribution of molecular weights. Estimated molecular weights of synthesized PTSgel were close to the feed ratio (Table 1).

Residual Tin, Organic Solvents, and Endotoxin Evaluation. Each polymer was tested for tin levels, organic solvents, 


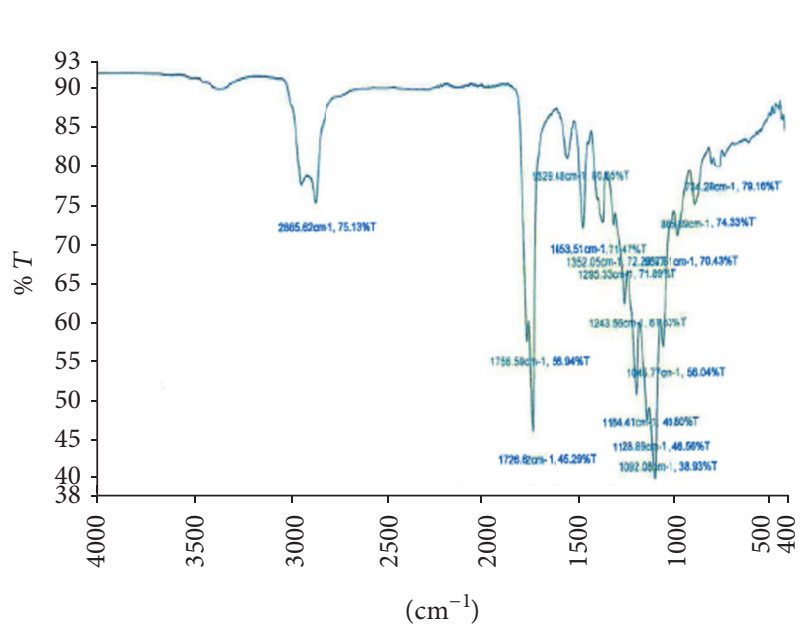

(a)

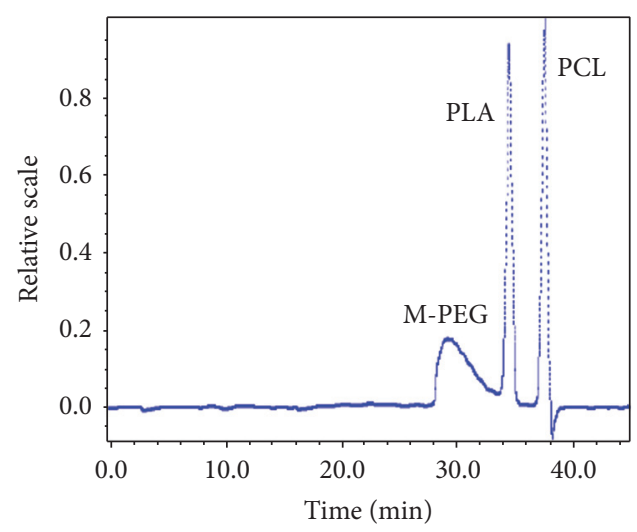

(c)

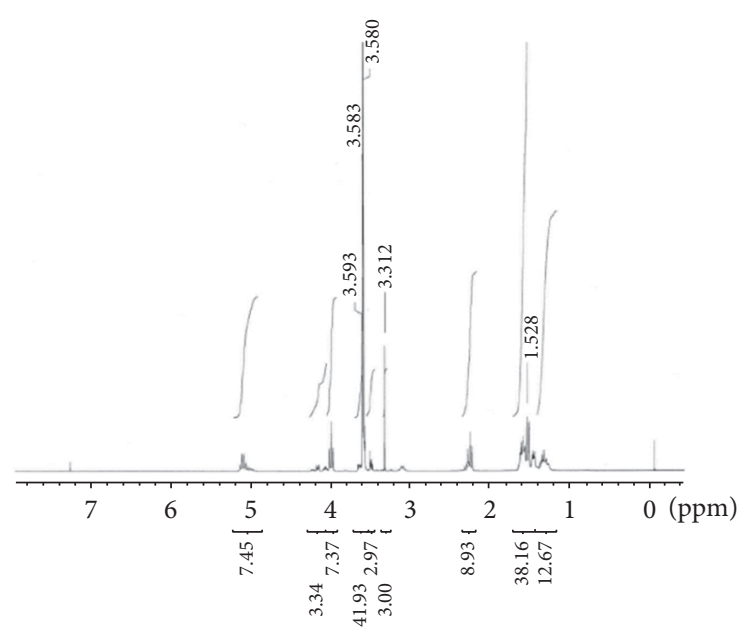

(b)

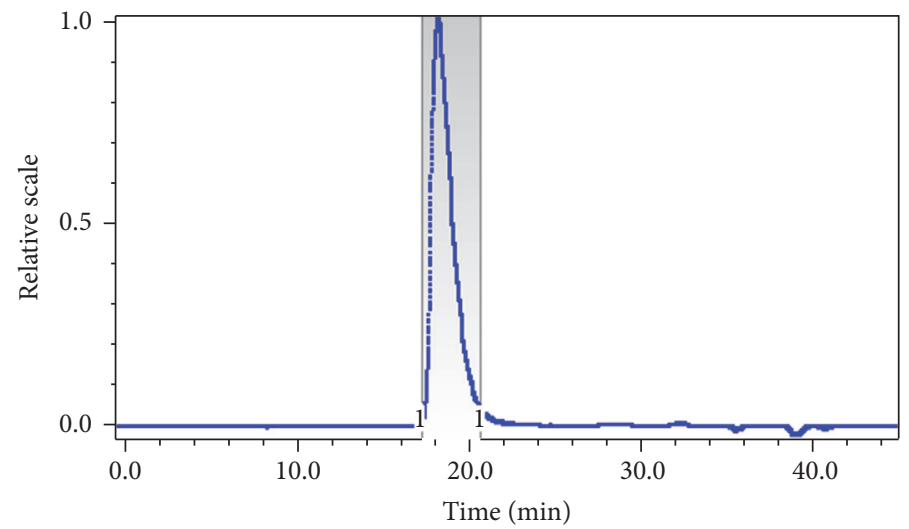

(d)

Figure 1: (a) FTIR spectrum of 10GH PTSgel. (b) ${ }^{1} \mathrm{H}$ NMR spectrum of $\mathrm{PEG}_{x}-\mathrm{PCL}_{y}-\mathrm{PLA}_{z}-\mathrm{PCL}_{y}-\mathrm{PEG}_{x}$. (c) GPC chromatogram for reference standards (m-PEG, PCL, and PLA). (d) GPC chromatogram for 10GH PTSgel. PD of the 10GH polymer $=1.08$.

and endotoxin levels. Sample analysis for the determination of residual dichloromethane (DCM) and heptane was performed by GC-MS. In all samples, no residual heptane was detected. Residual DCM was $0.2 \mathrm{ppm}(10 \mathrm{GH}), 124 \mathrm{ppm}$ (101GH), $74 \mathrm{ppm}(103 \mathrm{GH}), 170 \mathrm{ppm}(113 \mathrm{GH})$, and $278 \mathrm{ppm}$ (122GH). Stannous (tin) levels were less than $2 \mathrm{ppm}$. Endotoxin levels were determined to be less than $0.1 \mathrm{EU} / \mathrm{mL}$.

3.3. PTSgel Gelling Evaluation. All polymers were free flowing liquids at $4^{\circ} \mathrm{C}$. Sol-gel transition for the five polymers is as shown in Figure 2. The PTSgels 10GH and 103GH were clear liquids, whereas the PTSgels $113 \mathrm{GH}$ and $122 \mathrm{GH}$ were slightly turbid liquid at $4^{\circ} \mathrm{C}$ (Figure 2). Immediately after removal of the vials from the $37^{\circ} \mathrm{C}$ water bath, the PTSgels were a solid, slightly opaque white gel. On inversion of gel tubes at room temperature, the PTSgels remained a solid gel for 15 to 60 seconds (Figure 2). Eventually, all gels slowly transitioned back to liquid form at room temperature. All five polymers could be injected through a 31-gauge needle and form a solid gel in $\mathrm{PBS}$ at $37^{\circ} \mathrm{C}$ (see videos in Supplementary Material available online at http://dx.doi.org/10.1155/2016/2407459).
TABLE 1: Mw, Mn, and PDI determination of PTSgels.

\begin{tabular}{lccccc}
\hline PTSgel ID & $\begin{array}{c}\text { Total Mn }^{\mathrm{a}} \\
\text { (theoretical) }\end{array}$ & $\begin{array}{c}\text { Total } \mathrm{Mn}^{\mathrm{b}} \\
\text { (calculated) }\end{array}$ & $\begin{array}{c}\text { Total } \mathrm{Mn}^{\mathrm{c}} \\
\text { (calculated) }\end{array}$ & $\begin{array}{c}\mathrm{Mw}^{\mathrm{c}} \\
(\mathrm{GPC})\end{array}$ & $\mathrm{PDI}^{\mathrm{c}}$ \\
\hline $101 \mathrm{GH}$ & 3000 & 3898 & 4784 & 5132 & 1.07 \\
$10 \mathrm{GH}$ & 3100 & 3534 & 4855 & 5264 & 1.08 \\
$103 \mathrm{GH}$ & 3600 & 4271 & 3404 & 4347 & 1.28 \\
$113 \mathrm{GH}$ & 3500 & 3542 & 4615 & 5078 & 1.1 \\
$122 \mathrm{GH}$ & 3700 & 3941 & 4349 & 4941 & 1.14 \\
\hline
\end{tabular}

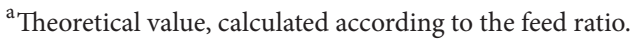

${ }^{\mathrm{b}}$ Calculated from ${ }^{1} \mathrm{H}$ NMR.

${ }^{\mathrm{c}}$ Determined by GPC analysis.

3.4. In Vitro PTSgel Disintegration (Gravimetric Measurement). The gravimetric dry weight loss over time of PTSgel 10GH $(12.5,18.75$, and $25 \%)$ in PBS was evaluated at $37^{\circ} \mathrm{C}$. There was a large difference in dry weight between the PTSgel concentrations at time 0, with 25\% polymeric gel having a mean weight of $120.8 \mathrm{mg}, 18.75 \%$ polymer of $79.1 \mathrm{mg}$, and $12.5 \%$ polymer of $41.8 \mathrm{mg}$. The lowest concentration 

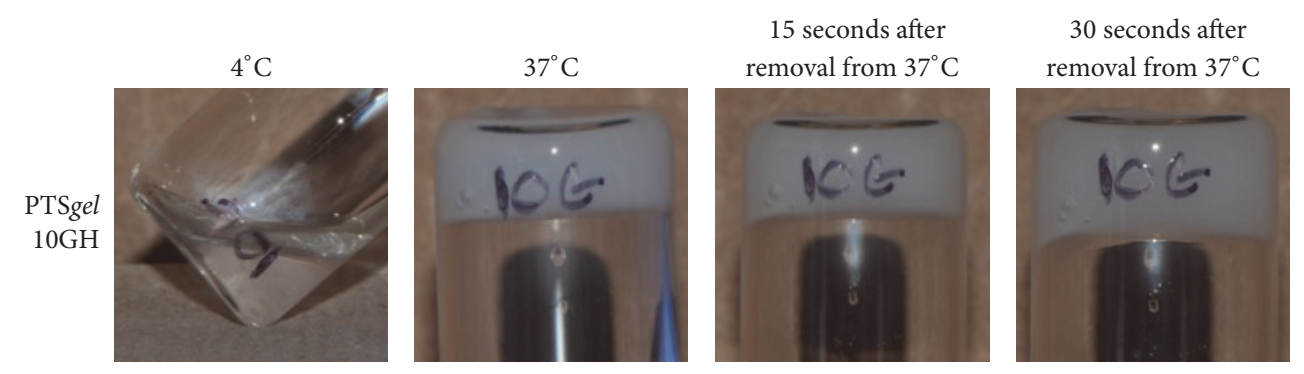

60 seconds after removal from $37^{\circ} \mathrm{C}$
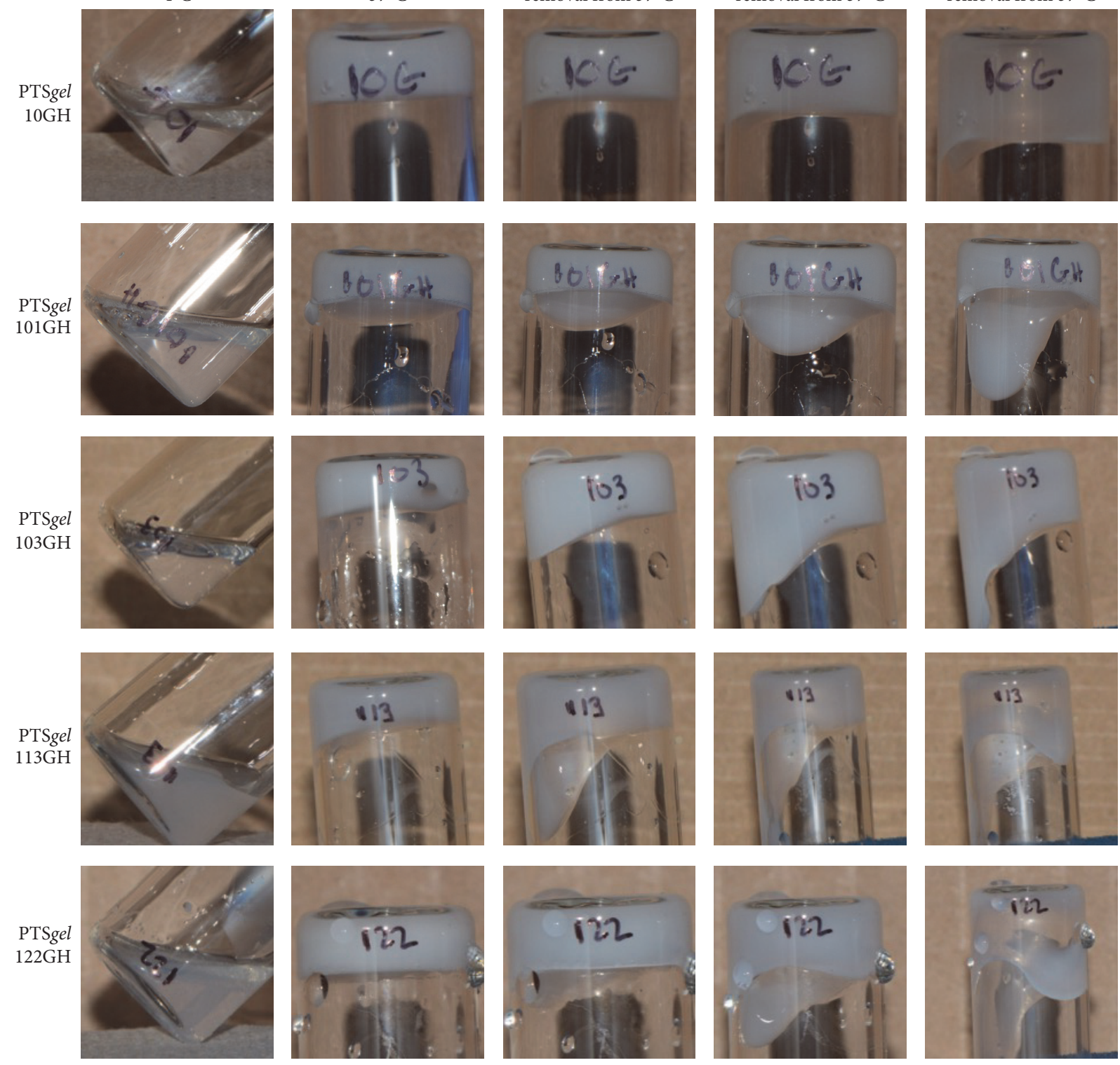

Figure 2: Sol-gel transition of $10 \mathrm{GH}, 101 \mathrm{GH}, 103 \mathrm{GH}, 113 \mathrm{GH}$, and $122 \mathrm{GH}$. At $4^{\circ} \mathrm{C}$ temperature the PTSgels are in liquid form and transition into solid gels at $37^{\circ} \mathrm{C}$. The gels slowly transition back to liquid form at room temperature.

of $10 \mathrm{GH}$ ( $12.5 \%$ gelling polymer) nearly completely disintegrated by 30 days (92.7\%), while 18.75 and 25\% 10GH PTSgel demonstrated disappearance of polymer with 74.2 and $76 \%$ dissolved, respectively, by day 75 (Figure 3(a)).

Residual polymer and supernatant collected at various time points were analyzed by GPC (Figure 3(b)). It is visually clear that the polymer peaks eluted as a symmetrical peak at day 0 and at days 30 and 60 . However, the peak at days 30 and 60 is wider than that observed at day 0 suggesting degradation of the polymer. The supernatant shows a small amount of dissolved polymers on day 0 ; however, there is no polymer present on day 30 and the disintegrated fragments are much smaller than the polymer but larger than the monomers used for the synthesis. The individual peaks have not yet been further characterized.

3.5. Modulation of In Vitro IgG Release from PTSgels. The solid gel PTSgel was maintained at $37^{\circ} \mathrm{C}$ and half of PBS buffer was removed and replaced on days 1, 2, 3, 4, 5, 7, 10 , and 14 and then weekly thereafter until no additional release was observed. Extensive modulation of in vitro release was achieved by using five different PTSgels, 101GH, 10GH, $103 \mathrm{GH}, 113 \mathrm{GH}$, and $122 \mathrm{GH}$, with increasing hydrophobicity of the polymers in the order, respectively. In most polymers, there was a low initial burst of drug release at $20 \mathrm{mg} / \mathrm{mL} \operatorname{IgG}$ $(11-24 \%$ release on day 1$)$ and an almost negligible release 


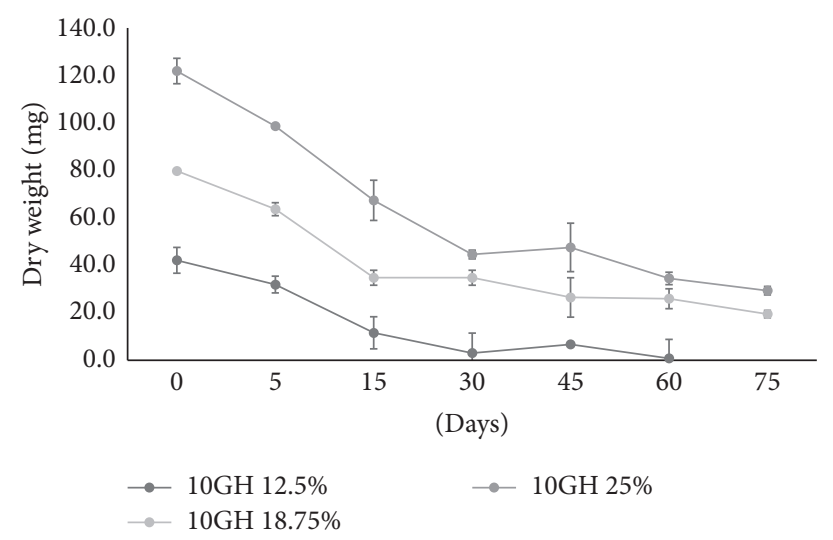

(a)
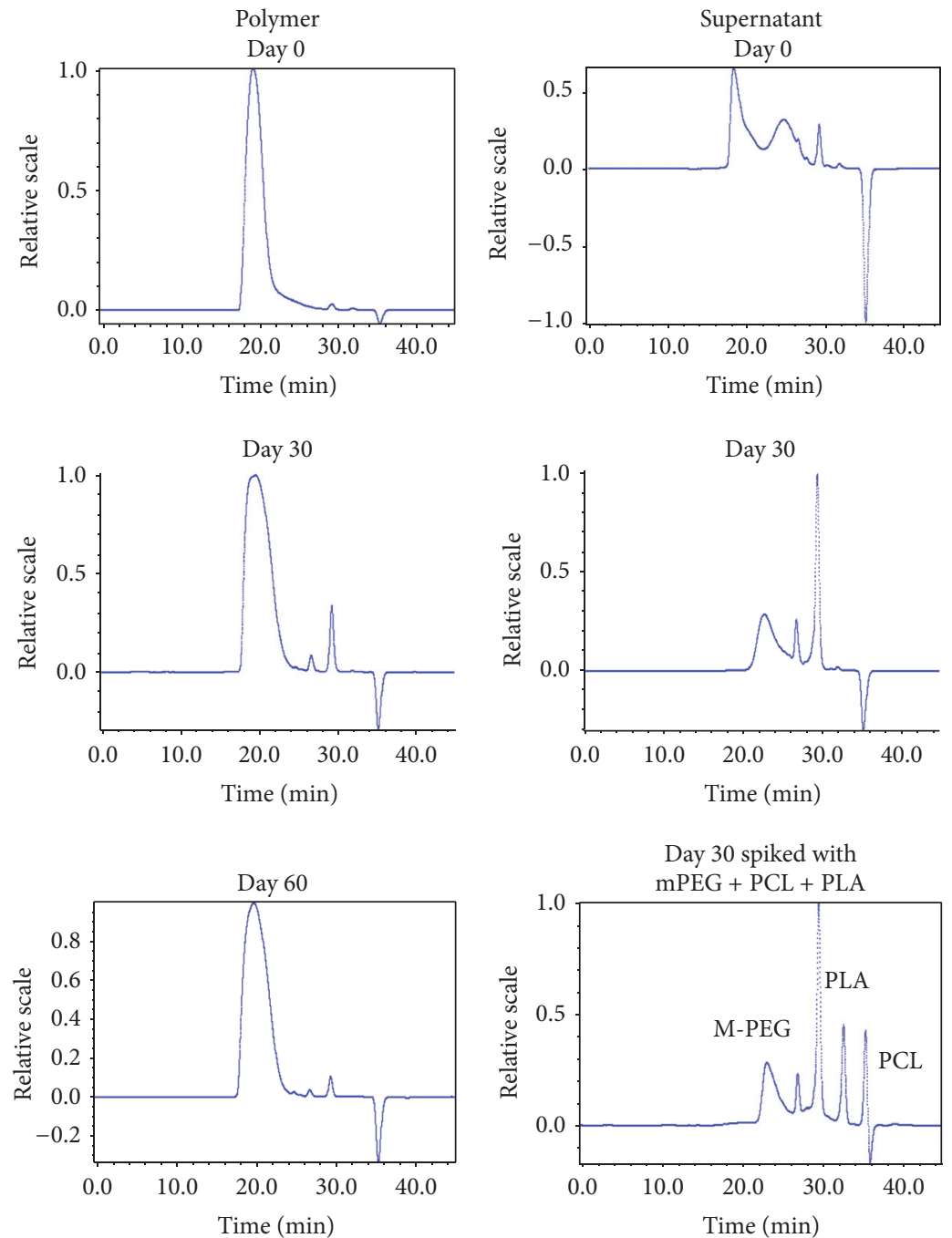

(b)

FIgURE 3: (a) In vitro dissolution/disintegration of $10 \mathrm{GH}$ PTSgel. Three concentrations $(12.5,18.75$, and $25 \%)$ of PTSgel $10 \mathrm{GH}(500 \mu \mathrm{L}$ in triplicate) were evaluated for disintegration in phosphate buffered saline ( $\mathrm{pH} 7.4$ ) in vitro at $37^{\circ} \mathrm{C}$. On day 0 and after $5,15,30$, and 45 days and every 15 days thereafter, overlaid buffer was removed and the remaining PTSgels were lyophilized and weighed to determine PTSgel dry weight over time. (b) GPC analysis of residual polymer (10GH, 25\%) and supernatant collected on various days for in vitro disintegration in PBS buffer, $\mathrm{pH} 7.4$ at $37^{\circ} \mathrm{C}$. 


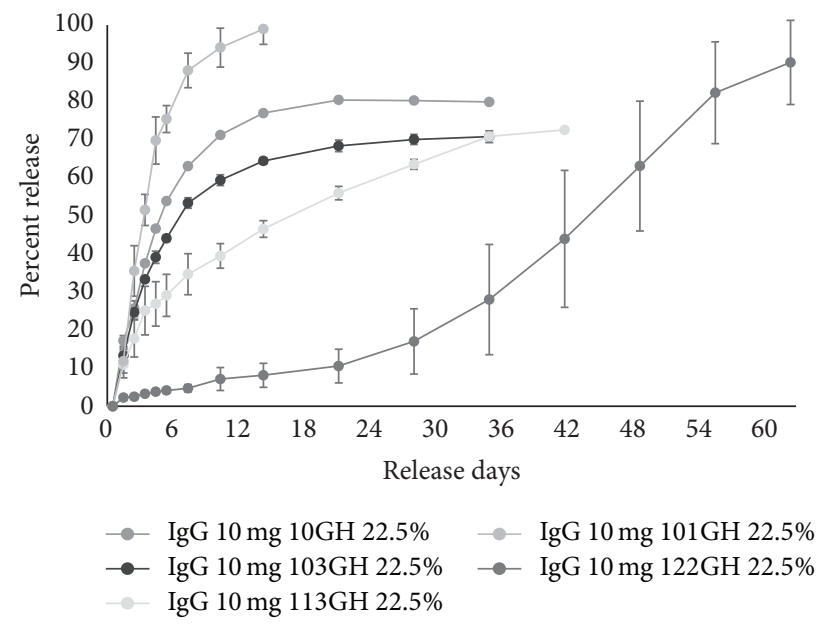

(a)

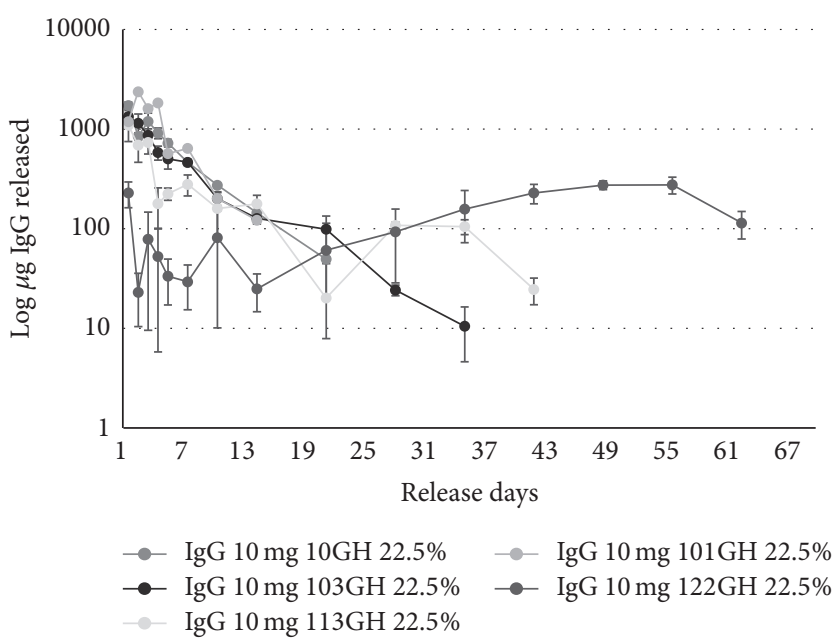

(b)

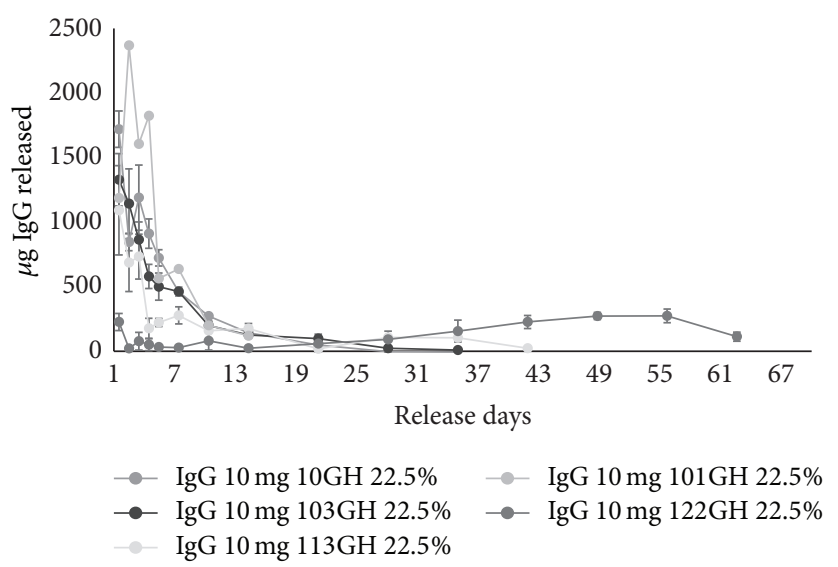

(c)

Figure 4: In vitro release of IgG from pentablock copolymers (PTSgel). Mean \pm SD of cumulative release (a) and daily release of IgG from PTSgel on a log scale (b) and on a linear scale (c). Using $20 \mathrm{mg} / \mathrm{mL}$ IgG in $22.5 \%$ PTSgel maintained at $37^{\circ} \mathrm{C}$, half of overlaid PBS buffer was removed and replaced at days $1,2,3,4,5,7,10$, and 14 and then weekly thereafter.

with $122 \mathrm{GH}(\sim 2 \%)$, the most hydrophobic polymer. Initial release is followed by a controlled release over extended period of several days to weeks as shown in Figure 4. Using $22.5 \%$ of each PTSgel, the quickest release rate was observed with the most hydrophilic polymer in the group (101GH) with $100 \%$ of the IgG load released in 14 days. The PTSgels $10 \mathrm{GH}$ and $103 \mathrm{GH}$ had a slower release with $80 \%$ and $69 \%$ of the IgG released in 21 days, respectively. Both the PTSgels $113 \mathrm{GH}$ and $122 \mathrm{GH}$ had a much slower rate of release with $73 \%$ and only $44 \%$ by day 42 , respectively. From 122GH, $91 \%$ of IgG release was achieved by day 63 (Figure 4(a)). A significant decrease in initial burst release related to increase in hydrophobicity demonstrates that the capacity to hold drugs possibly increases with increase in hydrophobicity of these PTSgels. Also, modulation of drug release at different rates can be achieved for long periods by changing the hydrophobicity of the polymers, as shown in Figures 4(b) and $4(\mathrm{c})$. As the polymer became more hydrophobic, the drug was released at a slower rate and the drug release had a much longer duration. The initial burst is also reduced considerably when lower $\operatorname{IgG}$ concentrations $(2 \mathrm{mg} / \mathrm{mL})$ were loaded into the polymer, as is shown in Figure 5 for PTSgel $10 \mathrm{GH}$, suggesting that the polymer's capacity to hold the drug may dictate initial burst release.

In addition, in vitro release of IgG was also demonstrated to be modulated by varying the concentration of PTSgel polymer. Using $2 \mathrm{mg} / \mathrm{mL}$ IgG in 10GH PTSgel, 9.6\% PTSgel resulted in a faster release of IgG, accompanied by a higher (40\%) initial release on day 1 and $80 \%$ cumulative release by day 7 , compared to a gradual and more sustained release achieved with 14.4 and $24 \%$ of $10 \mathrm{GH}$ PTSgel and less initial burst (Figure 5), again strongly suggesting that the drug release is a function of polymer capacity which increases with higher polymer concentration. Optimal loading for each therapeutic at different gelling polymer concentration will need to be determined during development.

\subsection{Structural Integrity of IgG Released from PTSgel}

(A) Using SDS-PAGE Analysis. Reduced (using betamercaptoethanol) and nonreduced sodium dodecyl sulfatepolyacrylamide gel electrophoresis (SDS-PAGE) were used 


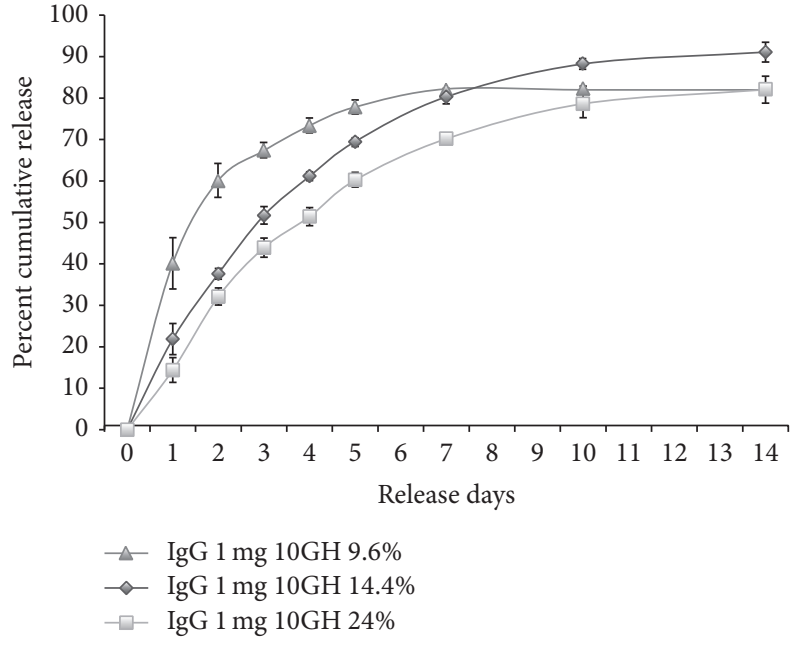

(a)

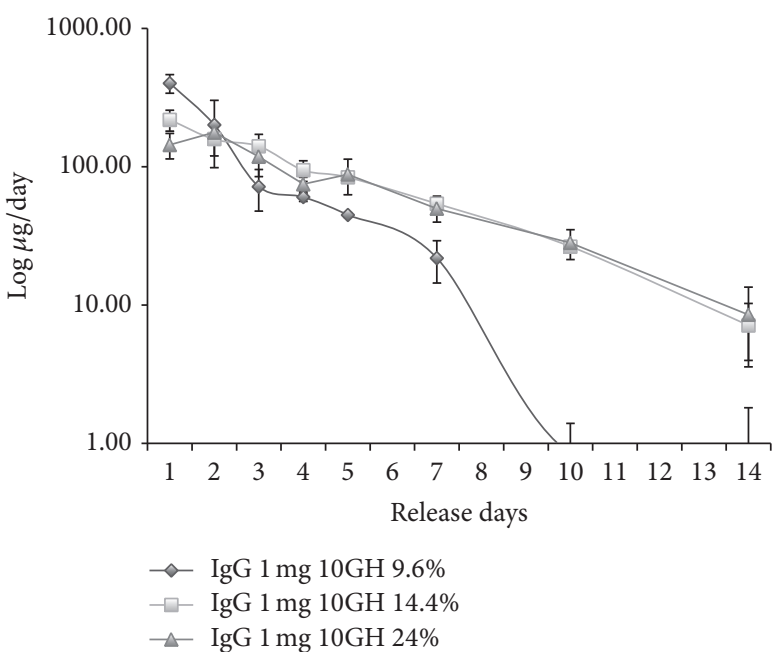

(b)

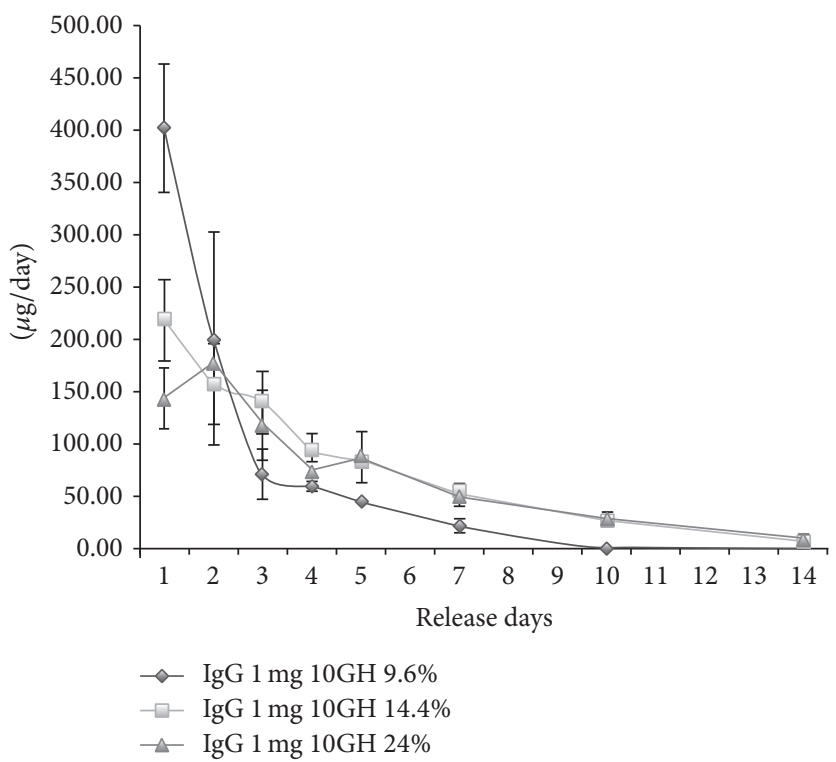

(c)

FIGURE 5: Effect of polymeric gel concentration on in vitro release of IgG from pentablock copolymers (PTSgel). Mean \pm SD of cumulative release (a) and $\log (\mathrm{b})$ and linear (c) daily release of IgG from PTSgel.

for size-based separations of the IgG for determining IgG integrity in the in vitro samples released from PTSgel over a period of seven days. Reducing conditions disrupt disulphide bonds separating IgG light and heavy chains. The IgG released from $10 \mathrm{GH}$ had the same bands of approximately $150 \mathrm{kDa}$ for the nonreduced and 28 and $51 \mathrm{kDa}$ for the reduced SDS-PAGE at days 1 and 7, which appeared identical to the IgG standards (Figure 6(a)). These results suggest that the IgG molecules were intact and no degradation of $\operatorname{IgG}$ had occurred during incubation with PTSgel and after release into the buffer. Similarly, there appeared to be excellent integrity for the IgG released from the $103 \mathrm{GH}$ polymer through 14 days (Figure 6(b)) and the 113GH polymer through 28 days (Figure 6(c)), suggesting that the PTSgel polymer did not affect the integrity of the
IgG protein. Low amounts of PLA utilized in the synthesis of PTSgel polymers do not result in lowering local $\mathrm{pH}$ upon polymer degradation and hence offer safe environment to drugs, especially biologics, for sustained release.

(B) SE-HPLC Analysis. Figures 6(d) and 6(e) compare chromatograms of SE-HPLC analysis conducted on a reference standard IgG and on an in vitro sample released after incubation with $10 \mathrm{GH}$ for 28 days at $37^{\circ} \mathrm{C}$. IgG maintained its structural integrity as is clearly demonstrated in Figures 6(d) and 6(e).

3.7. In Vivo Studies for IgG Release. Following subcutaneous injection of $200 \mu \mathrm{L}$ of PTSgel (either 10GH or 113GH) containing $200 \mu \mathrm{g}$ of near-infrared dye labeled IgG (NIR-IgG) or 


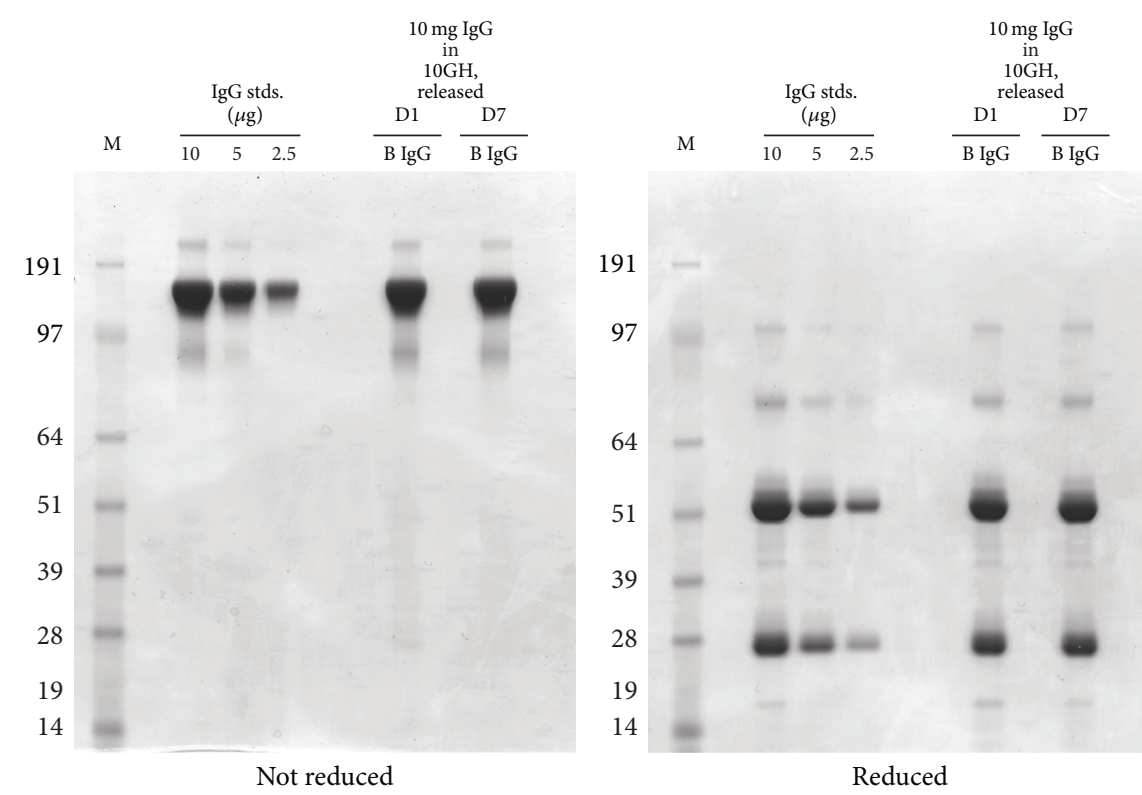

(a)
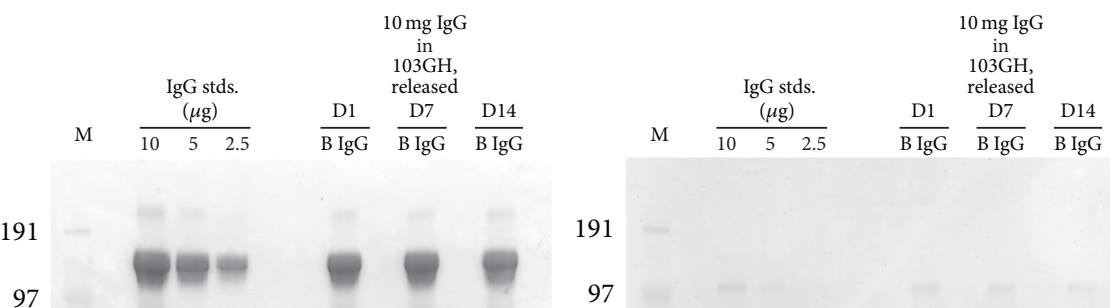

191

97

64

51

39

28

19

14

Not reduced

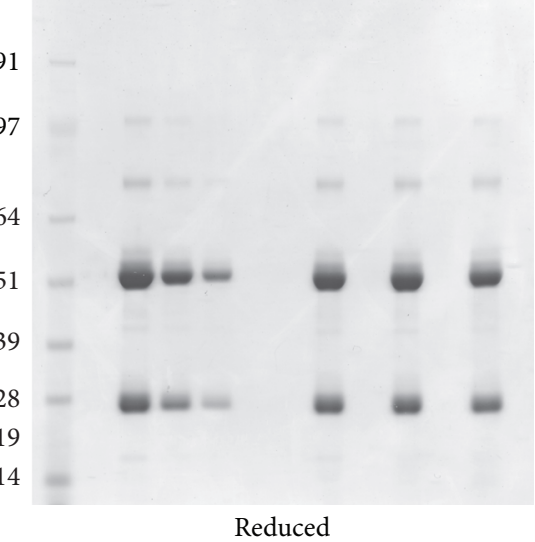

(b)

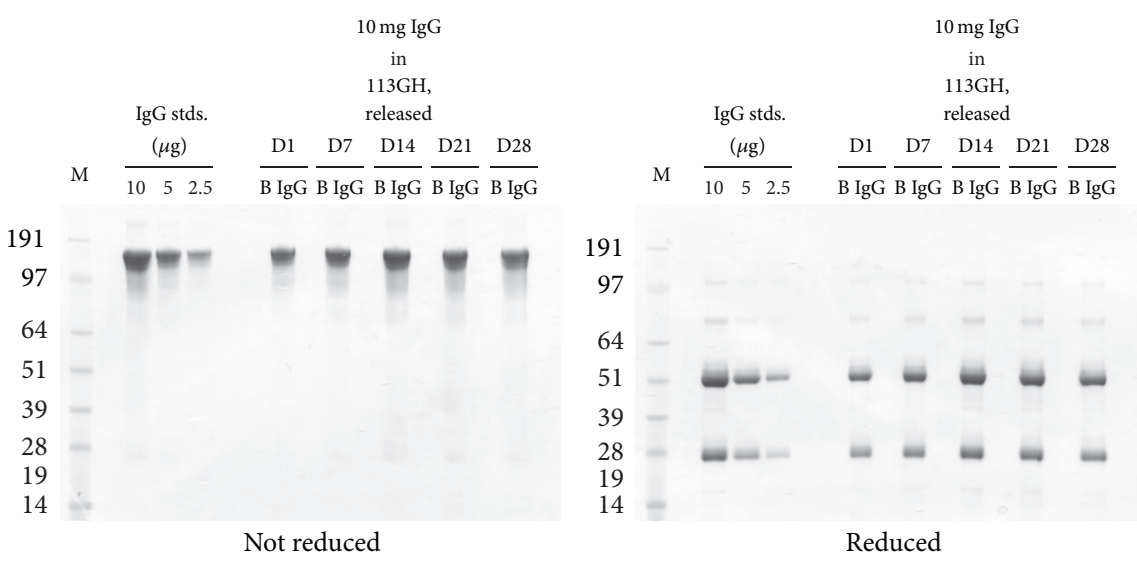

(c)

FIGURE 6: Continued. 


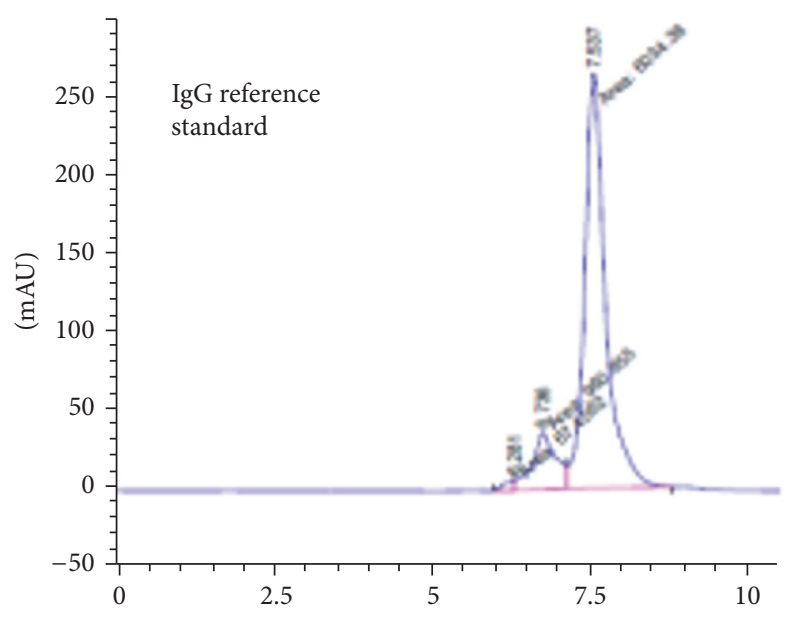

(d)

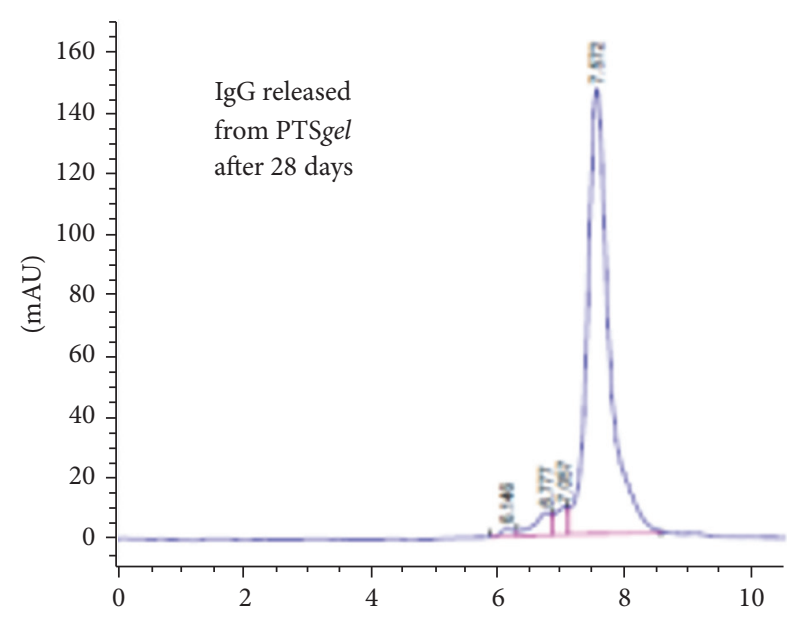

(e)

Figure 6: IgG integrity evaluated by SDS-PAGE. Reduced (using beta-mercaptoethanol) and nonreduced sodium dodecyl sulfatepolyacrylamide gel electrophoresis (SDS-PAGE) were used for size-based separations of the IgG for determining IgG integrity in the PTSgel in vitro release rate samples over time. The IgG released from $10 \mathrm{GH}$ (a), 103GH (b), and $113 \mathrm{GH}$ (c) had the same bands of approximately $150 \mathrm{kDa}$ for the nonreduced and 28 and $51 \mathrm{kDa}$ for the reduced SDS-PAGE at each time point tested, which appeared identical to the IgG standards. D, release day; B, blank lanes; M, molecular size marker; IgG stds., IgG standards. (d) and (e) SE-HPLC analysis of IgG reference standard (d) and of released sample (e) after incubation with PTSgel $10 \mathrm{GH}$ for 28 days in PBS at $37^{\circ} \mathrm{C}$.

NIR-IgG in PBS in mice, no adverse reaction, swelling, or redness was observed at the injection site for the duration of the study. NIR-IgG in PBS was visible on IVIS imaging immediately after injection but was no longer visible 24 hours after injection (Figures 7(a)-7(d)). Fluorescence of NIR-IgG in the PTSgel in vivo paralleled the in vitro release rates for $10 \mathrm{GH}$ and $113 \mathrm{GH}$ presented in Figure 4. Mice injected with $10 \% 10 \mathrm{GH}$ had fluorescence through approximately day 4, while those injected with $20 \% 10 \mathrm{GH}$ fluoresced through approximately day 14 (Figures 7(a) and 7(b)). Mice injected with $10 \% 113 \mathrm{GH}$ fluoresced through approximately 7 days, and those injected with 20\% 113GH fluoresced through approximately 35 days (Figures 7 (c) and 7(d)).

Once negative for fluorescence on IVIS imaging, mice were euthanized; the skin at the site of the injection was excised and imaged ex vivo. In all animals, there was a very small deposit of gel visible in the subcutaneous tissue, indicating the nearly complete dissolution/disintegration of the PTSgel (Figure 8). On imaging, there was a small signal of fluorescence that corresponded to approximately the size of the gel deposit, suggesting that IgG was tightly held by the PTSgel and that IgG release and gel dissolution/disintegration occurred in parallel and that an "empty shell" of undissolved PTSgel did not remain.

3.8. In Vivo Safety Assessment after Subcutaneous Injection of NIR-IgG with PTSgel. Following imaging, skin samples were fixed and processed for histopathology. When examined using light microscopy, the subcutaneous depot of PTSgel could be visualized and was mildly infiltrated with mononuclear cells. At the 6-day examination of the 10\% PTSgel 10GH, there were a few scattered neutrophils and macrophages surrounding and infiltrating the site of the injection. At 14 days with the $20 \%$ PTSgel, there were only a few macrophages associated with the gel deposit. At 42 days with the $113 \mathrm{GH}$ PTSgel, there were macrophages surrounding the depot but no epidermal or dermal inflammation or swelling was observed (Figure 9).

\section{Discussion}

We have demonstrated, both in vitro and in vivo, that PTSgels can modulate the release of IgG, a model therapeutic protein, from less than 7 days to greater than 63 days and possibly much longer by altering block composition for change in hydrophobicity and/or aqueous polymer solution concentration as well as amount of drug loaded in the polymer. These polymers seem to exhibit a very high capacity to hold IgG and thus result in minimal initial burst. The exact capacity for each polymer is currently undergoing investigation in our laboratory. We also demonstrated that the IgG was associated with the PTSgel as in vitro release and in vivo degradation of polymers occurred in parallel. In addition, we demonstrated that the PTSgel provides a safe environment for the protein as evidenced by the intact IgG structure when tested on day 28 of in vitro release from the PTSgel. Since IgG structure remained intact after release from PTSgel and based on work in progress in our laboratory, we anticipate that the functional proteins would also maintain their bioactivity when incubated with PTSgel. The PTSgel composition and concentration determined timing of gel dissipation in vivo. Only small remnants of PTSgel were present ex vivo in subcutaneous tissues of mice at the time of euthanasia and these gel remnants had NIR-labeled IgG visible on IVIS imaging, suggesting that the rate of $\operatorname{IgG}$ release and gel disappearance occurred in parallel. In a separate study, a similar phenomenon was observed 


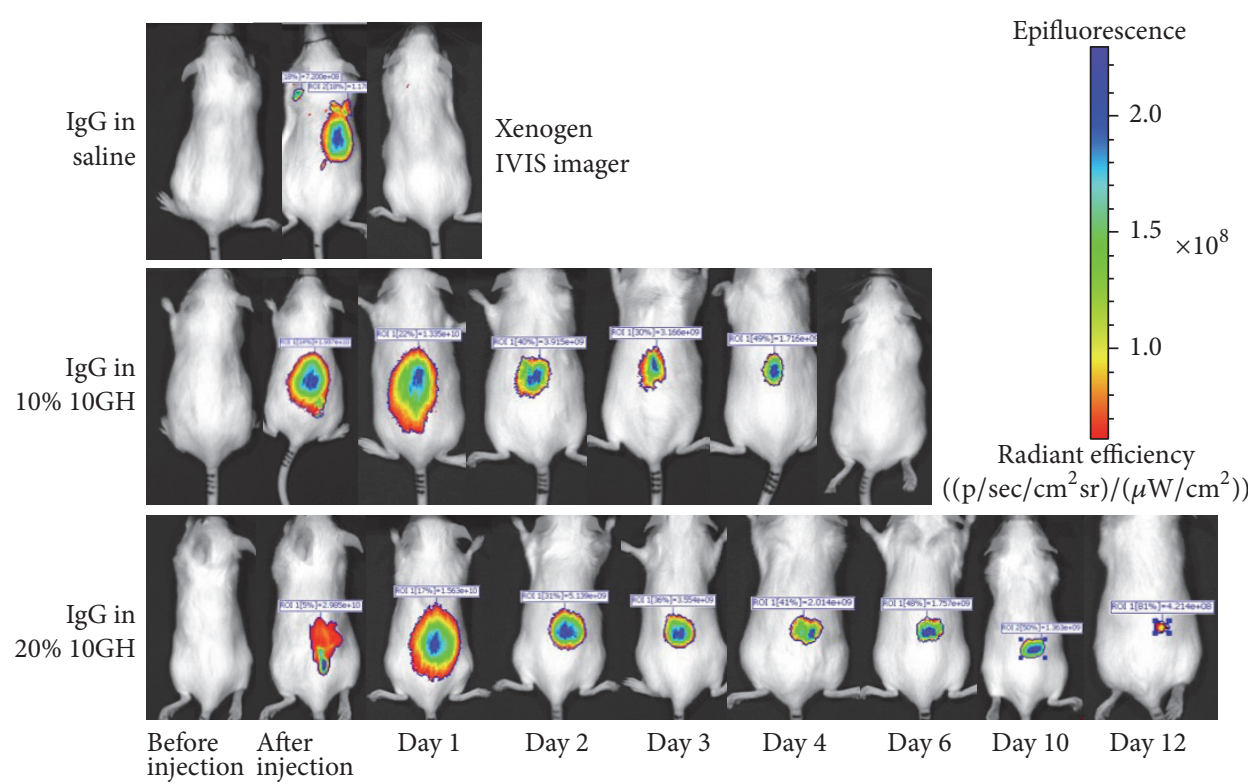

(a)

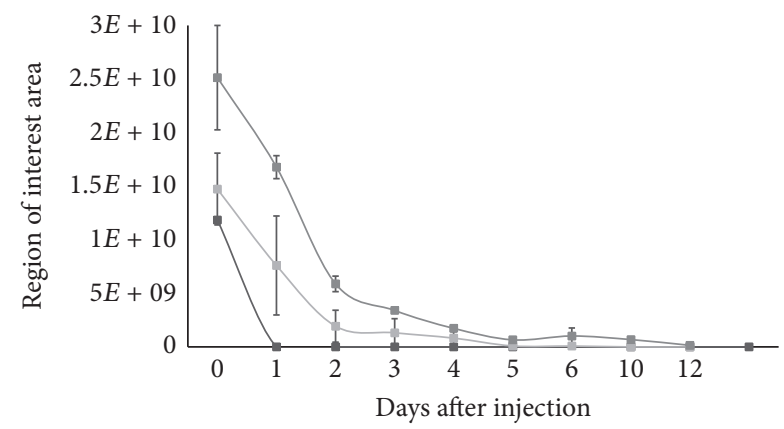

$\rightarrow$ NIR-IgG in BSS
$\rightarrow$ NIR-IgG in 10\% 10GH PTSgel

(b)

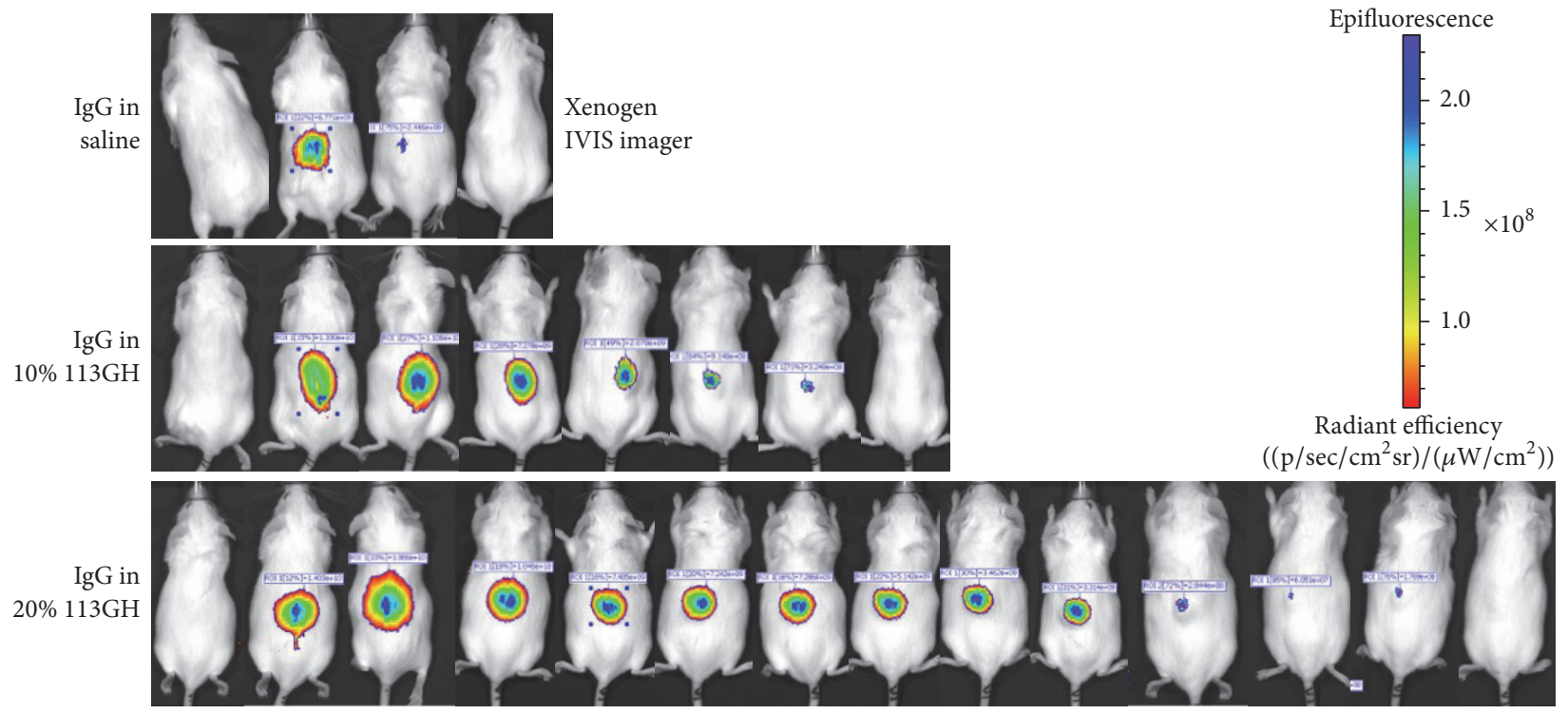

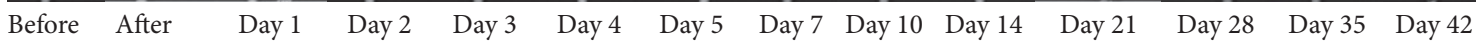
injection injection

(c)

FIGURE 7: Continued. 


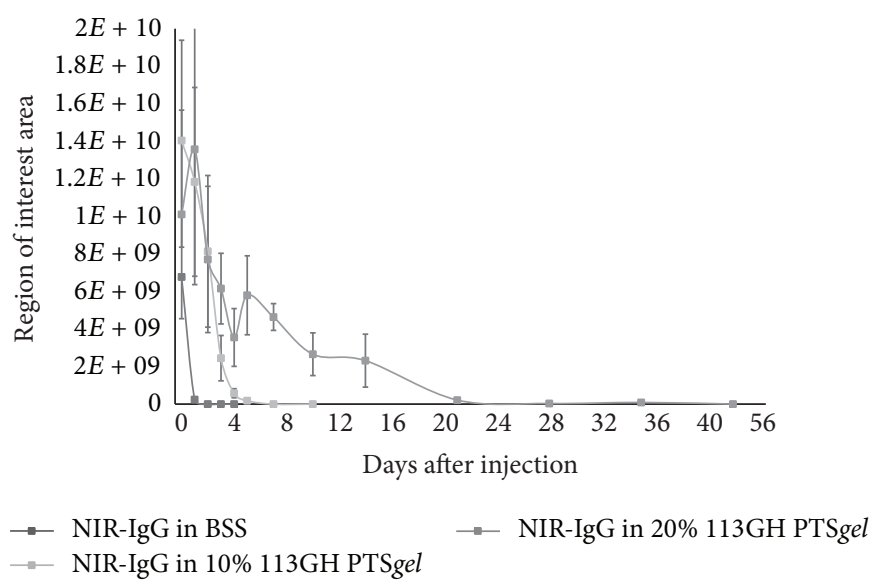

(d)

FIGURE 7: In vivo imaging of mice after subcutaneous injection of PTSgel. Mice were injected with PBS, PTSgel (10\% and 20\% 10GH or 113GH), or a combination of NIR-IgG PBS or NIR-IgG PTSgel in triplicate. Mice were anesthetized and imaged prior to injection, immediately after injection, and then on days 1-5, 7,10, and 14 after injection and then weekly thereafter using an in vivo imager (IVIS, Xenogen, Alameda, CA) $((\mathrm{a})$ and $(\mathrm{c}))$. Quantification of fluorescence was measured using the imaging software automatic region of interest (ROI) setting to calculate the radiant efficiency of the injection site ((b) and (d)).

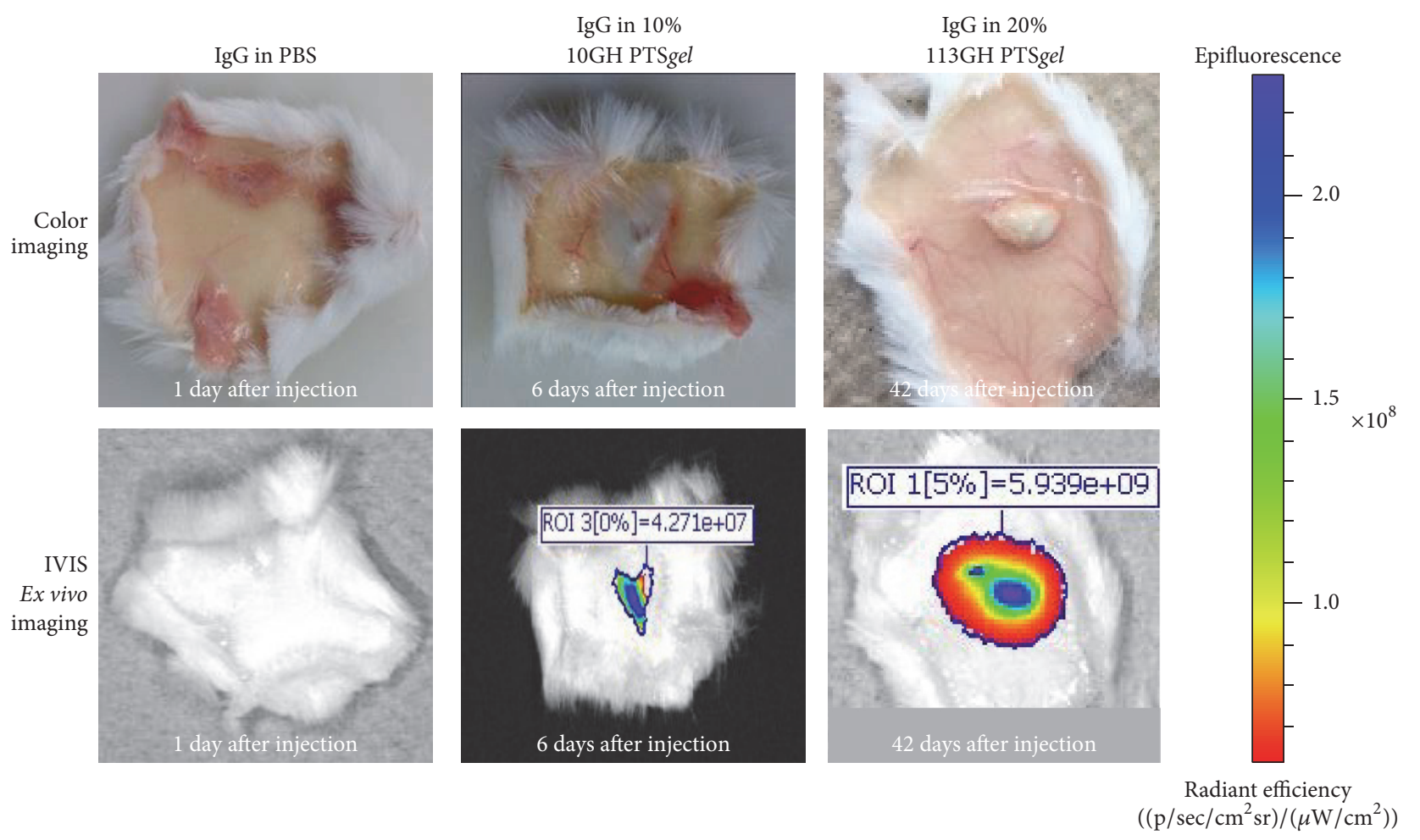

FIGURE 8: Ex vivo imaging of mice skin after subcutaneous injection of PTSgel. Once the mice were negative for fluorescence on IVIS imaging, they were euthanized; the skin at the site of the injection was excised and imaged ex vivo.

for NIR-labeled IgG when injected intracamerally [15]. The $25 \%$ PTSgel (10GH) gradually dissolved/disintegrated over the course of 30 days in the anterior chamber of the eye and the PTSgel had a strong signal of NIR-IgG in small remnants of PTSgel until the gel was completely dissolved or disintegrated [15].
When evaluating the in vitro IgG release, an initial 1day to 2-day higher release (burst effect) was observed with lower concentrations of PTSgel (9.6\% polymer), while in the higher concentrations of PTSgel (22 or $24 \%$ polymer), reduced burst effects were observed (see Figures 4-6) and they exhibited sustained release of IgG which paralleled the 


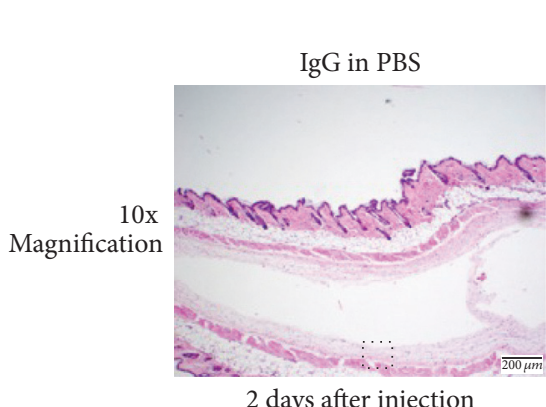

2 days after injection

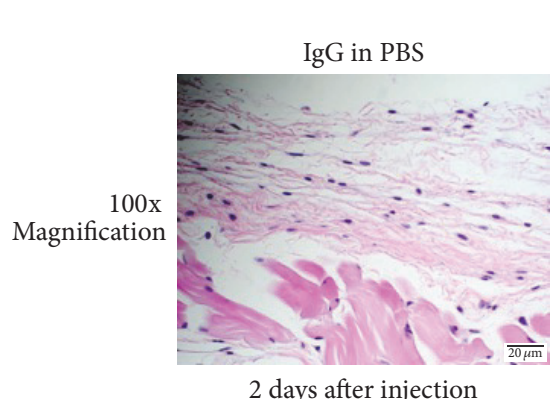

2 days after injection

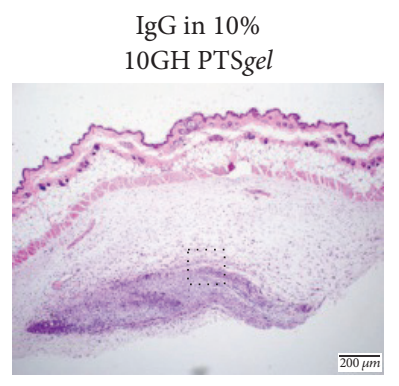

6 days after injection

(a)

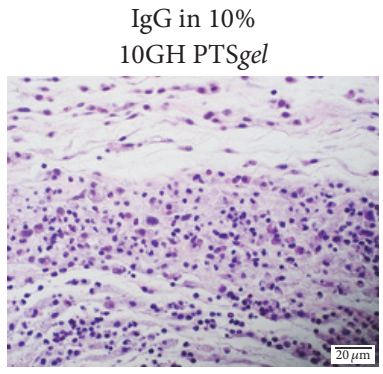

6 days after injection

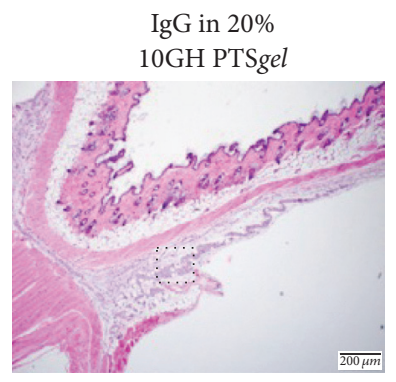

14 days after injection

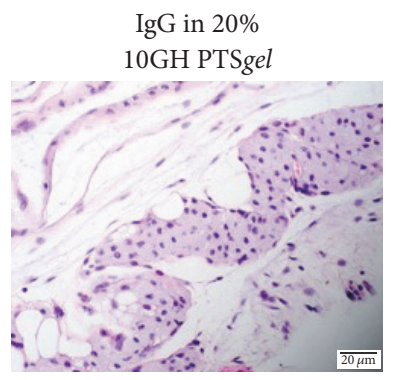

14 days after injection

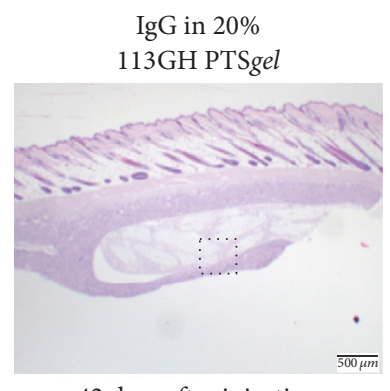

42 days after injection

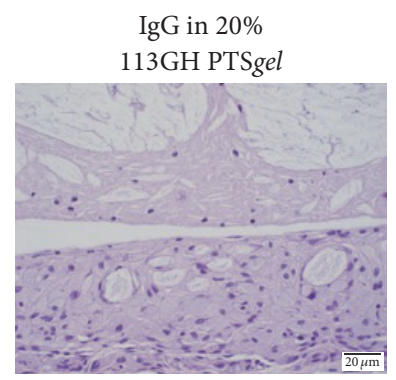

42 days after injection

(b)

Figure 9: Dermal histopathology after subcutaneous injection of PTSgel. Using lower magnification light microscopy (a), the subcutaneous depot of PTSgel could be visualized in the 10 and 20\% 10GH- and 113GH-injected tissue. Dotted box in (a) was the site of higher magnification represented in (b).

polymer dissolution/disintegration (see Figure 3), suggesting that the IgG was not just physically entrapped in the gel but perhaps a large portion of IgG is likely entrapped in the micellar structure of the polymer [12] which is released as the polymer dissolves or disintegrates. We have also observed this phenomenon with proteins other than IgG (data not shown). This suggests that initial burst effect likely occurs when added drug exceeds the capacity of the PTSgel to hold the drug for sustained release. As stated earlier, the dose capacity, though it has not been measured quantitatively, is likely dependent on a combination of factors such as polymer composition and percentage of the PTSgel.

In this study we achieved extensive modulation of $\operatorname{IgG}$ release from 7 days to longer than 63 days by varying the Mw size of PCL block comprising the PTSgel. Release rates of $\mathrm{IgG}$ from the fastest to the slowest (i.e., 101GH (fastest release) to $122 \mathrm{GH}$ (slowest release)) correlated with higher hydrophobicity created by increasing the PCL block size (from Mw 400 to 800). As shown in Figure 4, the PTSgel $122 \mathrm{GH}$ shows a continuing IgG release at 63 days, suggesting an expected duration of in vitro IgG release of 70 to 80 days. The PTSgel $113 \mathrm{GH}$ achieved sustained IgG release for at least 42 days (mean $278 \mu \mathrm{g}$ (day 7 ) to $25 \mu \mathrm{g}$ released on day 42 ), PTSgel 103GH for at least 21 days, and PTSgel 10GH for at least 14 days (each from a $22.5 \%$ polymer and total loading of $10 \mathrm{mg}$ IgG) (see Figure 4). Patel et al. [13] demonstrated similar release of IgG with PTSgel $113 \mathrm{GH}$ but required the use of polymer-IgG nanoparticles to achieve a 45-day release profile, which is far shorter duration than what we have been able to obtain with PTSgel 122GH alone.

Although we demonstrated in vivo that IgG remained in the gel until the polymer was nearly completely dissolved, further studies are needed to confirm drug bioactivity after delivery and to evaluate pharmacokinetics and pharmacodynamics for each route of therapy. However, a steady release in vitro which correlated well to the decrease in NIR-IgG in vivo was observed; hence one can hypothesize that in vivo drug release may be similar to that observed in vitro. Further studies are ongoing in our laboratory to evaluate drug bioactivity and to demonstrate pharmacokinetics and pharmacodynamics from protein and small molecule drug release of both hydrophobic and hydrophilic nature from a variety of PTSgels.

\section{Conclusions}

PTSgels create biocompatible/biodegradable thermosensitive formulations which can provide sustained release of biologics without chemical modification of the therapeutic molecule. Modulation of release and control of initial burst of IgG, a model therapeutic protein, was achieved by varying block composition and aqueous solution concentration of the PTSgels. When injected subcutaneously, PTSgels provided a safe environment for the protein and were well tolerated. 
Importantly, disintegration of the polymer paralleled IgG release in vivo, leaving little or no remnant of PTSgel after drug release was complete.

\section{Competing Interests}

Elizabeth Schaefer, Santhi Abbaraju, Mary Walsh, Donna Newman, and Jacklyn Salmon have no competing interests to declare. Rasidul Amin and Brian Gilger have financial interest in i-novion, Inc., and Ulrich Grau, Sidney Weiss, and Poonam Velagaleti are cofounders of i-novion, Inc.

\section{References}

[1] D. Chandler and A. Bewley, "Biologics in dermatology," Pharmaceuticals, vol. 6, no. 4, pp. 557-558, 2013.

[2] L. Kobrynski, "Subcutaneous immunoglobulin therapy: a new option for patients with primary immunodeficiency diseases," Biologics: Targets and Therapy, vol. 6, pp. 277-287, 2012.

[3] M. A. A. Siddiqui, "The efficacy and tolerability of newer biologics in rheumatoid arthritis: best current evidence," Current Opinion in Rheumatology, vol. 19, no. 3, pp. 308-313, 2007.

[4] J. G. Sathish, S. Sethu, M.-C. Bielsky et al., "Challenges and approaches for the development of safer immunomodulatory biologics," Nature Reviews Drug Discovery, vol. 12, no. 4, pp. 306-324, 2013.

[5] M. B. Atkins and M. Sznol, "Cancer immunotherapy: past progress and future directions," Seminars in Oncology, vol. 42, no. 4 , pp. 518-522, 2015.

[6] T. R. Hoare and D. S. Kohane, "Hydrogels in drug delivery: progress and challenges," Polymer, vol. 49, no. 8, pp. 1993-2007, 2008.

[7] R. Vaishya, V. Khurana, S. Patel, and A. K. Mitra, "Longterm delivery of protein therapeutics," Expert Opinion on Drug Delivery, vol. 12, no. 3, pp. 415-440, 2015.

[8] P. Agarwal and I. D. Rupenthal, "Injectable implants for the sustained release of protein and peptide drugs," Drug Discovery Today, vol. 18, no. 7-8, pp. 337-349, 2013.

[9] M. Qiao, D. Chen, X. Ma, and Y. Liu, "Injectable biodegradable temperature-responsive PLGA-PEG-PLGA copolymers: synthesis and effect of copolymer composition on the drug release from the copolymer-based hydrogels," International Journal of Pharmaceutics, vol. 294, no. 1-2, pp. 103-112, 2005.

[10] M. L. Houchin and E. M. Topp, "Chemical degradation of peptides and proteins in PLGA: a review of reactions and mechanisms," Journal of Pharmaceutical Sciences, vol. 97, no. 7, pp. 2395-2404, 2008.

[11] H. K. Makadia and S. J. Siegel, "Poly Lactic-co-Glycolic Acid (PLGA) as biodegradable controlled drug delivery carrier," Polymers, vol. 3, no. 3, pp. 1377-1397, 2011.

[12] S. P. Patel, R. Vaishya, X. Yang, D. Pal, and A. K. Mitra, "Novel thermosensitive pentablock copolymers for sustained delivery of proteins in the treatment of posterior segment diseases," Protein and Peptide Letters, vol. 21, no. 11, pp. 1185-1200, 2014.

[13] S. P. Patel, R. Vaishya, G. P. Mishra, V. Tamboli, D. Pal, and A. K. Mitra, "Tailor-made pentablock copolymer based formulation for sustained ocular delivery of protein therapeutics," Journal of Drug Delivery, vol. 2014, Article ID 401747, 15 pages, 2014.

[14] M. J. Hwang, J. M. Suh, Y. H. Bae, S. W. Kim, and B. Jeong, "Caprolactonic poloxamer analog: PEG-PCL-PEG," Biomacromolecules, vol. 6, no. 2, pp. 885-890, 2005.
[15] E. Schaefer, J. H. Salmon, S. Abbaraju et al., Sustained IgG Delivery to the Anterior Chamber of Rabbits Using Pentablock Co-polymers, ARVO Abstract, Association for Research in Vision and Ophthalmology, Seattle, Wash, USA, 2016. 

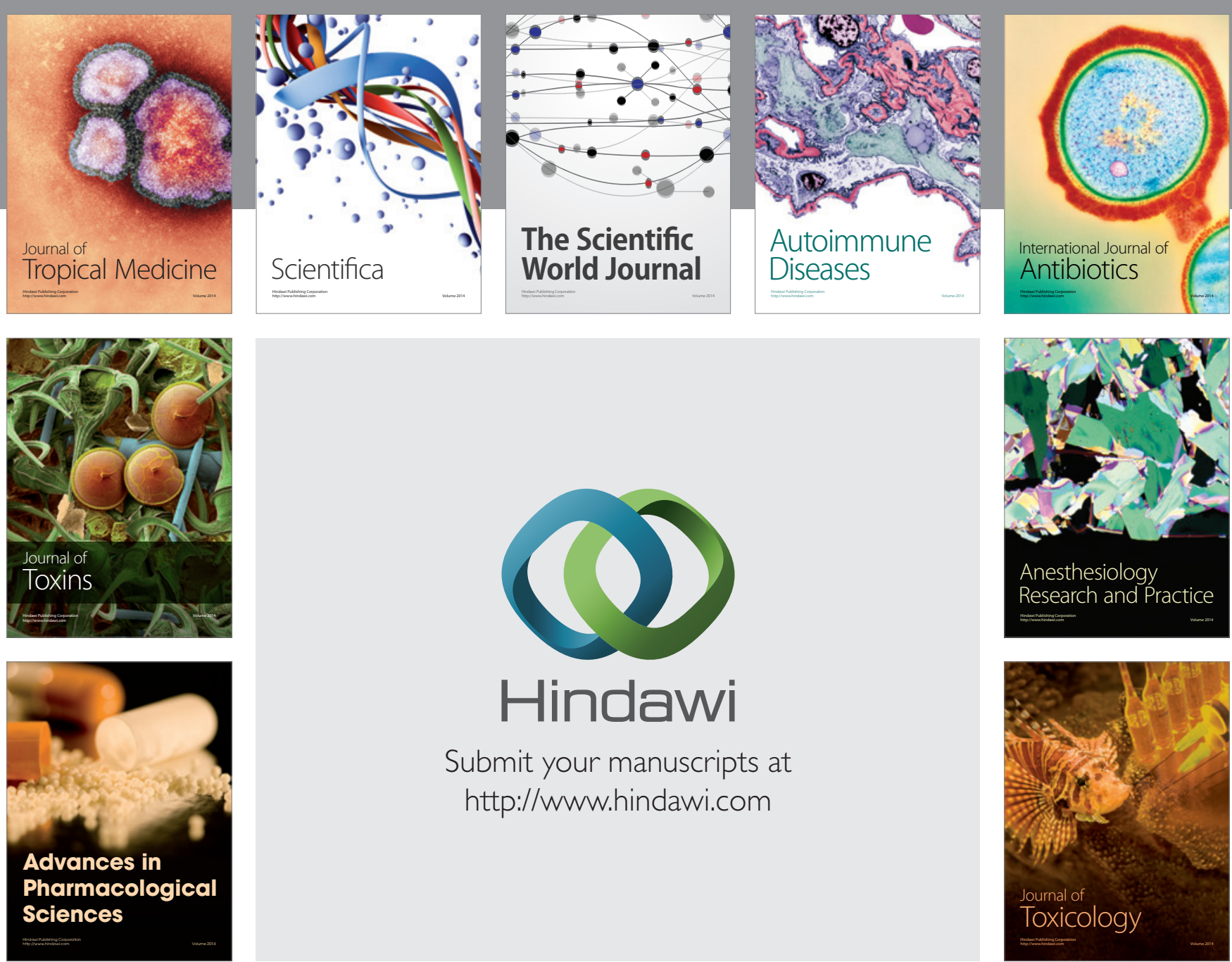

\section{Hindawi}

Submit your manuscripts at

http://www.hindawi.com
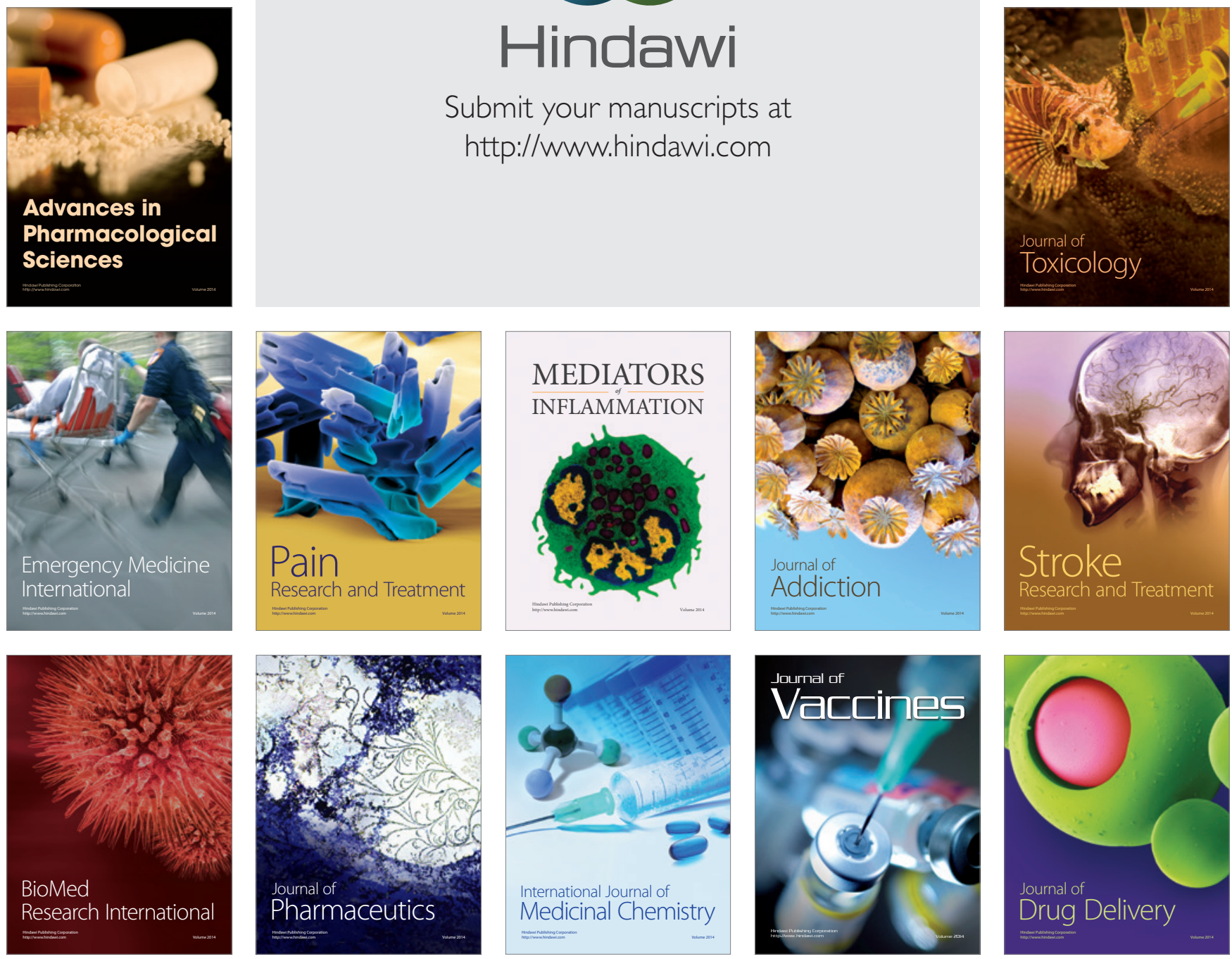